\title{
Trade Flows, Financial Linkage, and Business Cycles in Latin America
}

\author{
Magda Kandil \\ International Monetary Fund
}

\begin{abstract}
This paper studies co-movements in real output growth across countries of Latin America in the sample period 1970-2007. To detect the change over time, correlations in real growth are analyzed over two sub-samples: 1970-1986 and 1987-2007. Correlation coefficients detect co-movements in real growth across five regional groups: Andean, Caribbean, Central America, LAC7(Argentina, Brazil, Chile, Columbia, Mexico, Peru, Venezuela) and Mercosur. In addition, growth in each of the EU, the U.S., and Brazil are assumed to be common sources of co-movements in real output growth in Latin America. Business-cycle comovements within and across regions have varied over time. We also examine the impact of trade flows and financial linkage on co-movements in real growth within and across regions. The effect of trade flows is not conclusive. Business cycles may diverge, converge, or not vary significantly in response to trade flows across countries. More recently, co-movement in real growth appears to be tracking comovement in short-term interest rates across Latin America.
\end{abstract}

- JEL Classification: E32, F41, F42, F15, O10

- Keywords: Business Cycle Synchronization, Linkages, Bilateral Trade Flows, Spill-over Effects, Specialization, LDCs, Intra-industry Trade, Integration

\footnotetext{
*Corresponding address: Magda Kandil: International Monetary Fund 700 nineteenth Street, N.W. Washington D.C., 20431 Tel: (202)589-4996, Fax: (202)589-4996, E-mail: mkandil@imf.org. (C2011-Center for Economic Integration, Sejong Institution, Sejong University, All Rights Reserved.
} 


\section{Introduction}

The nineties marked an era of globalization that coincided with deliberate efforts to achieve a higher degree of trade and financial liberalization. Countries that have subscribed to this plan have opened up their borders to trade and financial flows. While many countries have capitalized on the benefits of trade and financial integration, liberalization carries the potential risk of being exposed to external shocks. As the world economy has become more integrated, a downturn in one economy spreads faster to another. Increased interdependence means that much of the world can move down in tandem. Hence is the interest to study co-movements in real growth across countries of Latin America.

Trade linkages may affect the demand and supply sides of a given economy. On the demand side, imports may support higher demand for consumption and investment. Higher investment may contribute to an increase in the output supply. In addition, export growth may have a direct positive effect on real growth. Through these types of spill-over effects, stronger trade linkages can result in more highly correlated business cycles. However, stronger trade linkages may be indicative of a higher degree of specialization across countries. Hence, higher trade flows would be consistent with industry-specific shocks in exporting countries that dominate the business-cycle effect of trade flows, decreasing the degree of synchronization of business cycles across trading partners (see Frenkel and Rose, 1998).

The effects of financial linkages are also uncertain on co-movements in real growth. More financial integration could result in large wealth effects as capital migrates from exporting countries to importing countries. Through this channel, the positive spill-over effects are transmitted from capital abundant countries to capital scarce countries. This scenario could result in a higher degree of business-cycle comovement through financial linkages. Nonetheless, financial linkages may decrease cross-country output co-movement. For example financial linkages may stimulate specialization of production through the allocation of capital in a manner consistent with countries' comparative advantage. ${ }^{1}$

In a world of increased financial and trade integration, recent empirical studies have embarked on analyzing the impact of these linkages on co-movements in business cycles. Some studies employ cross-country or cross-region panel

${ }^{1}$ See, e.g., Kalemli-Ozcan, Sorensens and Yosha (2003). 
regression to assess the role of global linkages. ${ }^{2}$ Others, see, e.g., Imbs (2004a, 2004b) have documented the positive effects of financial integration on crosscountry output and consumption correlation. ${ }^{3}$ Co-movements in business cycles with trade and financial linkages may shift over time in response to a shift in the underlying determinants of these linkages. For example, the increased wave of capital flows and financial integration in the nineties may have resulted in a shift in the evolution of business-cycle synchronization.

A number of studies have tracked shifts in linkages across countries over time. Helbling and Bayoumi (2003) find that correlation coefficients between the United States and other Group of Seven (G-7) countries for the period 1973-2001 are substantially lower than those for 1973-89. ${ }^{4}$ Similarly, Stock and Watson (2003) analyze the change in co-movement between business cycles in the G-7 countries. They conclude that common shocks are less important more recently (1982-2002), compared to the earlier sample period (1960-83). In contrast, other studies have contradicted this finding. Kose, Prasad, and Terrones (2003) study the correlation between the fluctuations in individual country aggregates (output, consumption, and investment) and those in corresponding World (G-7) aggregates. Their results support an increase in correlations over time.

More recent studies have employed factor models to establish the importance of common factors in driving the degree of business-cycle co-movement. Kose, Otrok, and Whiteman (2003) establish an important role for common factors in explaining business cycles in developed countries, relative to developing countries. Along the same lines, Gregory, Head, and Raynald (1997) use Kalman filtering techniques to identify common fluctuations across macroeconomic aggregates using dynamic factor model.

In the spirit of the above investigations, this paper examines co-movements in real output growth across major regional blocks of Latin America: Andean, ${ }^{5}$

\footnotetext{
${ }^{2} \mathrm{~A}$ number of recent studies have demonstrated positive effects of trade linkages on business-cycle comovement. See, e.g., Frankel and Rose (1998), Clark and van Wincoop (2001) and Kose and Yi (2005). Similarly, Baxter and Kouparitsas (2004) and Otto, Voss and Willard (2003) document that international trade is the most important channel of business cycles.

${ }^{3}$ In support of both trade and financial linkages is the evidence in Kose, Prasad and Terrones (2003).

${ }^{4}$ Heathcate and Perri (2002) document changes in the correlation of output, consumption, and investment between the United States and an aggregate of Europe, Canada, and Japan over time. In contrast, Doyle and Faust (2003) indicate no significant change in the correlations between the growth rate in the United States and other G-7 countries over time.

${ }^{5}$ Group of Andean: Bolivia, Colombia, Ecuador, Peru and Venezuela.
} 
Caribbean, ${ }^{6}$ Central America, ${ }^{7}$ LAC $7,{ }^{8}$ and Mercosur. ${ }^{9}$ Of particular interest is to study co-movements in real growth in individual countries underlying each regional group and the aggregate of real growth in other regional blocks. ${ }^{10}$ In addition, three major shocks are considered as common factors and introduced into all correlation exercises for the various regional groups in Latin America. The first common source is growth in the U.S. The second source is growth in the EU and the third source is growth in Brazil. In the first step of the analysis, the paper measures co-movements in real growth across regions of Latin America and with growth in the common sources. The second step analyzes the impacts of trade and financial linkages, across regional blocks and with the common sources, on comovements in real output growth.

Four objectives underlie the paper's analysis. First, is to provide evidence on the strength of co-movements in real growth across regions of Latin America. Second, is to study the impact of growth in three common sources, the U.S., the EU, and Brazil, on business cycles in Latin America over time. Third, is to provide evidence on the impact of trade and financial linkages on co-movements in business cycles across regions in Latin America and with the common sources. Fourth, is to study the evolution of co-movements in real growth over time in two sub-periods: 1970-1986 and 1987-2006. The latter period has marked an increase in globalization and, therefore, a surge in intra regional trade and financial flows. ${ }^{11}$

\footnotetext{
${ }^{6}$ Group of Caribbean: Caricom Group, Dominican Republic and the Netherlands Antilles. Caricom Group: ECCU, the Bahamas, Barbados, Belize, Guyana, Haiti, Jamaica, Suriname, and Trinidad \& Tobago. ECCU Group: Antigua \& Barbuda, Dominica, Grenada, St. Lucia, St. Kitts \& Nevis, and St. Vincent \& the Grenadines.

${ }^{7}$ Group of Central America: Costa Rica, El Salvador, Guatemala, Honduras, Nicaragua, and Panama.

${ }^{8}$ Group of LAC7: Argentina, Brazil, Chile, Colombia, Mexico, Peru, and Venezuela.

${ }^{9}$ Group of Mercosur: Argentina, Brazil, Chile, Paraguay, and Uruguay.

${ }^{10}$ Given overlap between members in some groups, the common country is excluded from growth in other regional growth to study cross-regional correlation.

${ }^{11}$ The truncation of the sample period is guided by evidence in previous studies outlining steps taken by the group of countries under investigation to lower trade impediments among themselves in the latter period, compared to the earlier period. In addition, financial barriers have been falling steadily in many of these countries starting in late eighties and throughout the nineties. In addition, developments in trade shares (see below) support the hypothesis of structural break.
} 


\section{Data and Methodology}

The analysis employs data of real output growth in five regional blocks of Latin America, Andean, Caribbean, Central America, LAC7, and Mercosur. ${ }^{12}$ In addition, the analysis incorporates real growth in individual country members of each regional group. Further, the analysis includes real growth in three common sources of business cycles in Latin America, the U.S., EU, and Brazil.

Real GDP in dollar in 2005 is constructed using nominal GDP in current prices in dollar and the GDP deflator. Real GDP in constant dollar prices is constructed by applying the real GDP growth rates backward. Real GDP for regional groups is a weighted geometric average of GDP across member countries with weights that correspond to each country's share of the regional GDP.

Trade linkage is measured as the value of exports and imports relative to GDP. These linkages are computed for member countries in each regional block in Latin America with other regional blocks and the common sources. Financial linkage is measured by bilateral co-movements in short-term nominal deposit interest rates between member countries and with common sources. ${ }^{13}$

To measure co-movements in real growth, the analysis is based on the Pearson correlation coefficients. ${ }^{14}$ By construction, real growth is the first difference of the $\log$ value of real GDP that measures cyclical fluctuations around a stochastic trend over time. ${ }^{15}$ When variables are highly correlated, their points tend to fall on or near a line of fit. If the variables are negatively correlated, the line of fit has a negative slope.

\footnotetext{
${ }^{12}$ Data description and sources are provided in Appendix A.

${ }^{13}$ The interest rate is endogenous to domestic conditions and external flows. For example, countries that have a common currency peg, e.g., to the US dollar (or highly dollarized economies) are likely to align the interest rate with movements in the US Federal Fund rate, increasing co-movements among their interest rates. The less financially integrated countries are the more likely domestic priorities would guide movements in the interest rate. In contrast, under financial liberalization, movements in capital flows would be attracted to the interest rate differential, increasing destabilizing pressures on foreign reserves and/or the exchange rate. To mitigate the latter effects, countries adjust interest rate policies in response to financial flows, narrowing the difference across interest rates with respect to financially integrated economies.

${ }^{14}$ For details, see Noether (1967) and Knight (1966).

${ }^{15}$ As noted in the seminal work of Nelson and Plosser (1982), economic series are characterized by nonstochastic trends, requiring first-differencing to render the series stationary. The resulting series is the domain of business cycles. In contrast, measuring deviations around a deterministic trend may over- or under-estimate the business cycle component.
} 
Table 1. Summary Statistics: Real GDP Growth

\begin{tabular}{ccccc}
\hline \multirow{2}{*}{ Country Groups and Major Countries } & \multicolumn{2}{c}{$1987-2006$} & \multicolumn{2}{c}{$1970-1986$} \\
\cline { 2 - 5 } & Mean & Std. Dev. & Mean & Std. Dev. \\
\hline EU & 2.34 & 1.01 & 2.77 & 1.81 \\
United States & 3.06 & 1.24 & 3.07 & 2.71 \\
Brazil & 2.21 & 2.37 & 6.01 & 5.05 \\
LAC7 & 2.6 & 2.0 & 4.5 & 3.5 \\
Andean & 2.5 & 3.2 & 3.6 & 3.1 \\
Caribbean & 3.2 & 2.0 & 2.0 & 2.5 \\
Central America & 3.7 & 2.9 & 2.7 & 2.6 \\
Mercosur & 2.5 & 2.3 & 4.7 & 4.1 \\
\hline
\end{tabular}

Andean: Bolivia, Colombia, Ecuador, Peru, Venezuela.

Caribbean: Antigua and Barbuda, The Bahamas, Barbados, Belize, Dominica, Dominican Republic, Grenada, Guyana, Haiti, Jamaica, Netherlands Antilles, St. Lucia, St. Kitts \& Nevis, St. Vincent \& The Grenadines, Suriname, Trinidad \& Tobago.

Central America: Costa Rica, El Salvador, Guatemala, Honduras, Nicaragua, Panama.

LAC7: Argentina, Brazil, Chile, Colombia, Mexico, Peru, Venezuela.

Mercosur: Argentina, Brazil, Chile, Paraguay, Uruguay.

\section{Analysis of Results}

Table 1. summarizes the mean and standard deviation of real growth in the three common sources and the five regional blocks of Latin America in the two subperiods under investigation.

Except for the Caribbean and Central America, average real growth is lower in the various regional blocks in the latter period, 1987-2007. Except for the Andean Group and Central America, the variability of real growth has decreased over time. Across the common sources, growth is relatively comparable in the two subperiods in each of the EU and the U.S.

The highest trade shares in the most recent period are within Central America, followed by Caricom, Caribbean, Mercosur, LAC7, the Andean Group and the ECCU. Further, there has been a surge in trade relations starting 1987 within most regional blocks. The highest trade relation shares for the U.S. are with LAC7, which is dominated by trade relations between the U.S. and Mexico.

Table $2 \mathrm{a}, \mathrm{b}$ presents correlations between real growth across the five regional blocks in Latin America and with growth in the three common sources. Correlation coefficients are contrasted between the earlier sample period 1970-86 and the latter sample period 1987-2007.

Contrary to the hypothesis of increased integration, correlations between real 
Table 2a. Co-Movements in Real GDP Growth Sample Period: 1970-1986

\begin{tabular}{lcccccccc}
\hline $\begin{array}{l}\text { Country } \\
\text { Group }\end{array}$ & U.S. & EU & Brazil & $\begin{array}{c}\text { Andean - } \\
\text { Self }\end{array}$ & Caribbean & $\begin{array}{c}\text { Central } \\
\text { America }\end{array}$ & $\begin{array}{c}\text { LAC7 } \\
\text { - Self }\end{array}$ & $\begin{array}{c}\text { Mercosur } \\
\text { - Self }\end{array}$ \\
\hline LAC7 & 0.19 & $0.64^{*}$ & $0.96^{*}$ & $0.67^{*}$ & $0.60^{*}$ & $0.50^{*}$ & & $0.47^{*}$ \\
& $(0.47)$ & $(0.0076)$ & $(0.0001)$ & $(0.0043)$ & $(0.015)$ & $(0.05)$ & & $(0.064)$ \\
Andean & 0.0004 & 0.38 & $0.73^{*}$ & & $0.46^{* *}$ & $0.55^{*}$ & $0.73^{*}$ & $0.71^{*}$ \\
& $(0.99)$ & $(0.15)$ & $(0.0014)$ & & $(0.07)$ & $(0.029)$ & $(0.0012)$ & $(0.0023)$ \\
Caribbean & -0.034 & $0.52^{*}$ & $0.52^{*}$ & $0.46^{* *}$ & & 0.23 & $0.60^{*}$ & $0.54^{*}$ \\
& $(0.90)$ & $(0.038)$ & $(0.039)$ & $(0.07)$ & & $(0.40)$ & $(0.015)$ & $(0.029)$ \\
Central & 0.33 & $0.50^{*}$ & $0.51^{*}$ & $0.55^{*}$ & 0.23 & & $0.50^{*}$ & $0.47^{* *}$ \\
America & $(0.22)$ & $(0.05)$ & $(0.043)$ & $(0.029)$ & $(0.40)$ & & $(0.05)$ & $(0.067)$ \\
Mercosur & 0.24 & $0.69^{*}$ & $0.97^{*}$ & $0.71^{*}$ & $0.54^{*}$ & $0.47^{* *}$ & $0.51^{*}$ & \\
& $(0.38)$ & $(0.003)$ & $(0.0001)$ & $(0.0023)$ & $(0.029)$ & $(0.067)$ & $(0.04)$ & \\
\hline
\end{tabular}

Table 2b. Co-Movements in Real GDP Growth Sample Period: 1987-2006

\begin{tabular}{lcccccccc}
\hline $\begin{array}{l}\text { Country } \\
\text { Group }\end{array}$ & U.S. & EU & Brazil & $\begin{array}{c}\text { Andean } \\
\text {-Self }\end{array}$ & Caribbean & $\begin{array}{c}\text { Caribbean } \\
\text { America }\end{array}$ & $\begin{array}{c}\text { LAC7 } \\
\text {-Self }\end{array}$ & $\begin{array}{c}\text { Mercosur } \\
\text { - Self }\end{array}$ \\
\hline LAC7 & 0.30 & -0.15 & $0.80^{*}$ & $0.69^{*}$ & 0.26 & 0.38 & & $0.97^{*}$ \\
& $(0.22)$ & $(0.53)$ & $(0.0001)$ & $(0.0012)$ & $(0.28)$ & $(0.11)$ & & $(0.0001)$ \\
Andean & -0.10 & -0.30 & $0.40^{* *}$ & & 0.18 & 0.38 & $0.56^{*}$ & $0.61^{*}$ \\
& $(0.69)$ & $(0.21)$ & $(0.09)$ & & $(0.47)$ & $(0.11)$ & $(0.012)$ & $(0.005)$ \\
Caribbean & 0.24 & 0.12 & 0.25 & 0.18 & & 0.29 & 0.26 & 0.22 \\
& $(0.32)$ & $(0.63)$ & $(0.31)$ & $(0.47)$ & & $(0.23)$ & $(0.28)$ & $(0.35)$ \\
Central & -0.073 & $-0.49^{*}$ & 0.04 & 0.38 & 0.29 & & 0.38 & 0.27 \\
America & $(0.77)$ & $(0.035)$ & $(0.87)$ & $(0.11)$ & $(0.23)$ & & $(0.11)$ & $(0.26)$ \\
Mercosur & 0.28 & -0.15 & $0.88^{*}$ & $0.61^{*}$ & 0.22 & 0.27 & $0.46 *$ & \\
& $(0.25)$ & $(0.53)$ & $(0.0001)$ & 0.005 & $(0.35)$ & $(0.26)$ & $(0.047)$ & \\
\hline
\end{tabular}

growth in the EU and growth in Latin America were larger and significant in the earlier sample period, compared to the latter period. It is worth noting, however, that co-movements in real growth in Latin America with the U.S. are not significant over time, despite geographical proximity.

Across regions of Latin America, the spillover effects from Brazil were more significant in the earlier period in the Caribbean and Central America. For the remaining blocks, significant co-movements with real growth in Brazil are larger in 1970-86, compared to 1987-2007.

In contrast to the implications of higher integration in the latter period, comovements in real growth appear, in general, stronger in the earlier period, compared to the latter period. This applies to co-movements across regions of Latin America and between these regions and growth in the common sources: the 


\section{U.S, EU, and Brazil. ${ }^{16}$}

To shed additional light on cross-regional correlations, the analysis turns to real growth in individual countries within regional groups.

\section{A. Andean group}

Table 3. summarizes the mean and standard deviation in real growth across member countries of the Andean group.

Except for Bolivia, average real growth is generally lower in the latter period, compared to the earlier period. Except for Peru and Venezuela, fluctuations are less severe in 1987-2007, compared to 1970-86.

Except for the U.S., co-movements in real growth in Andean countries varied

Table 3. Summary Statistics: Real GDP Growth

\begin{tabular}{lcccc}
\hline & \multicolumn{2}{c}{$1987-2006$} & \multicolumn{2}{c}{$1970-1986$} \\
\hline Andean Group & Mean & Std. Dev. & Mean & Std. Dev. \\
\hline Bolivia & 3.5 & 1.33 & 2.31 & 3.92 \\
Colombia & 3.3 & 2.3 & 4.82 & 2.44 \\
Ecuador & 3.0 & 3.8 & 6.42 & 6.20 \\
Peru & 2.4 & 6.2 & 3.25 & 4.94 \\
Venezuela & 2.5 & 6.8 & 3.09 & 3.95 \\
\hline
\end{tabular}

Source: World Economic Outlook.

Table 4a. Co-Movements in Real GDP Growth Sample Period: 1970-1986

\begin{tabular}{lcccccccc}
\hline $\begin{array}{l}\text { Andean } \\
\text { Group }\end{array}$ & U.S. & EU & Brazil & $\begin{array}{c}\text { LAC7 - Andean } \\
\text { Self }\end{array}$ & $\begin{array}{c}\text { Self } \\
\text { Caribbean }\end{array}$ & $\begin{array}{c}\text { Central } \\
\text { America }\end{array}$ & Mercosur \\
\hline Bolivia & 0.016 & $0.52^{*}$ & $0.50^{*}$ & $0.64^{*}$ & $0.61^{*}$ & $0.57^{*}$ & $0.68^{*}$ & $0.55^{*}$ \\
& $(0.95)$ & $(0.03)$ & $(0.041)$ & $(0.007)$ & $(0.013)$ & $(0.022)$ & $(0.004)$ & $(0.026)$ \\
Colombia & 0.22 & $0.73^{*}$ & $0.51^{*}$ & $0.74^{*}$ & $0.43^{* *}$ & $0.45^{* *}$ & $0.64^{*}$ & $0.71^{*}$ \\
& $(0.40)$ & $(0.0009)$ & $(0.036)$ & $(0.0011)$ & $(0.094)$ & $(0.078)$ & $(0.007)$ & $(0.0019)$ \\
Ecuador & 0.34 & $0.68^{*}$ & $0.65^{*}$ & $0.63^{*}$ & $0.51^{*}$ & $0.49^{*}$ & $0.53^{*}$ & $0.56^{*}$ \\
& $(0.19)$ & $(0.004)$ & $(0.007)$ & $(0.009)$ & $(0.05)$ & $(0.052)$ & $(0.03)$ & $(0.024)$ \\
Peru & -0.29 & 0.043 & $0.46^{* *}$ & $0.52^{*}$ & $0.45^{* *}$ & 0.21 & 0.23 & $0.51^{*}$ \\
& $(0.26)$ & $(0.87)$ & $(0.064)$ & $(0.04)$ & $(0.08)$ & $(0.44)$ & $(0.40)$ & $(0.05)$ \\
Venezuela & -0.026 & $0.52^{*}$ & $0.44^{* *}$ & $0.51^{*}$ & $0.61^{*}$ & $0.51^{*}$ & $0.61^{*}$ & $0.50^{*}$ \\
& $(0.92)$ & $(0.03)$ & $(0.08)$ & $(0.046)$ & $(0.012)$ & $(0.04)$ & $(0.011)$ & $(0.046)$ \\
\hline
\end{tabular}

Source: World Economic Outlook.

Magnitudes in parentheses indicate the probability of zero correlation.

$*$ and $* *$ denote significance at the five and ten $\%$ levels.

\footnotetext{
${ }^{16}$ This evidence supports the findings of Helbling and Bayoumi (2003) and Stock and Watson (2003).
} 
Table 4b. Average Shares of Trade ((Exports + Imports)/GDP) with Major Trading Partners (in \%) Sample Period: 1970-1986

\begin{tabular}{lcccccccc}
\hline \multirow{2}{*}{ Group of Andean } & U.S & EU & Brazil & $\begin{array}{c}\text { Andean- } \\
\text { Self }\end{array}$ & $\begin{array}{c}\text { Carib- } \\
\text { bean }\end{array}$ & $\begin{array}{c}\text { Central } \\
\text { America }\end{array}$ & $\begin{array}{c}\text { LAC7 }- \\
\text { Self }\end{array}$ & $\begin{array}{c}\text { Merco- } \\
\text { sur }\end{array}$ \\
\hline Bolivia & 12.93 & 11.14 & 3.25 & 1.74 & 0.001 & 0.08 & 15.93 & 14.3 \\
Colombia & 6.88 & 6.43 & 0.32 & 1.63 & 0.34 & 0.37 & 2.33 & 0.9 \\
Ecuador & 15.51 & 3.58 & 0.18 & 2.75 & 15.34 & 0.27 & 3.8 & 0.31 \\
Peru & 7.85 & 6.25 & 0.74 & 1.53 & 0.13 & 0.2 & 2.9 & 1.79 \\
Venezuela & 15.24 & 7.4 & 0.89 & 0.66 & 3.95 & 0.86 & 2.3 & 1.5 \\
\hline
\end{tabular}

Source: Direction of Trade Statistics.

significantly with the EU, Brazil, and other regional blocks in Latin America. Table 4a illustrates co-movements in real growth in 1970-1986 between members of the Andean and other regional blocks, excluding self, as well as correlations with real growth in the common sources. Despite significant co-movements of real output growth in Colombia, the country's trade linkages are generally small in Table $4 \mathrm{~b}$. In general, trade linkages do not correspond to significant co-movements in real growth.

Overall, co-movements are less pronounced in the latter period between real growth in members of the Andean group and growth in the common sources and other regional blocks. Table 5a, b replicates the evidence for Andean countries over the latter sample period, 1987-2007. Most notably are the insignificant comovements with growth in the EU, Brazil, and the Caribbean, compared to

Table 5a. Co-Movements in Real GDP Growth Sample Period: 1987-2006

\begin{tabular}{llccccccc}
\hline $\begin{array}{l}\text { Andean } \\
\text { Group }\end{array}$ & U.S. & EU & Brazil & $\begin{array}{c}\text { LAC7- } \\
\text { Self }\end{array}$ & $\begin{array}{c}\text { Andean- } \\
\text { Self }\end{array}$ & $\begin{array}{c}\text { Caribbean } \\
\text { America }\end{array}$ & Mercosur \\
\hline Bolivia & -0.06 & -0.04 & 0.17 & $0.41^{* *}$ & $0.41^{* *}$ & 0.041 & 0.28 & 0.37 \\
& $(0.79)$ & $(0.86)$ & $(0.47)$ & $(0.08)$ & $(0.08)$ & $(0.87)$ & $(0.24)$ & $(0.12)$ \\
Colombia & -0.05 & -0.16 & 0.32 & $0.41^{* *}$ & $0.42^{* *}$ & -0.09 & 0.034 & $0.47^{*}$ \\
& $(0.82)$ & $(0.50)$ & $(0.17)$ & $(0.08)$ & $(0.07)$ & $(0.71)$ & $(0.89)$ & $(0.04)$ \\
Ecuador & -0.17 & -0.06 & -0.03 & 0.21 & $0.46^{*}$ & 0.29 & $-0.40^{* *}$ & 0.19 \\
& $(0.47)$ & $(0.79)$ & $(0.90)$ & $(0.39)$ & $(0.05)$ & $(0.23)$ & $(0.09)$ & $(0.44)$ \\
Peru & 0.014 & -0.30 & $0.56^{*}$ & $0.54^{*}$ & 0.19 & $0.39^{* *}$ & $0.47^{*}$ & $0.60^{*}$ \\
& $(0.95)$ & $(0.19)$ & $(0.011)$ & $(0.018)$ & $(0.45)$ & $(0.10)$ & $(0.04)$ & $(0.006)$ \\
Venezuela & -0.04 & 0.017 & 0.034 & 0.31 & 0.36 & -0.12 & 0.16 & 0.28 \\
& $(0.86)$ & $(0.94)$ & $(0.89)$ & $(0.20)$ & $(0.13)$ & $(0.62)$ & $(0.51)$ & $(0.25)$ \\
\hline
\end{tabular}

Source: World Economic Outlook.

Magnitudes in parenthesis indicate the probability of zero correlation.

$*$ and $* *$ denote significance at the five and ten $\%$ levels. 
Table 5b. Average Shares of Trade ((Exports + Imports)/GDP) with Major Trading Partners (in percent) Sample Period: 1987-2006

\begin{tabular}{lcccccccc}
\hline $\begin{array}{l}\text { Group of } \\
\text { Andean }\end{array}$ & U.S. & EU & Brazil & $\begin{array}{c}\text { Andean } \\
\text { Self }\end{array}$ & Caribbean & $\begin{array}{c}\text { Central } \\
\text { America }\end{array}$ & $\begin{array}{c}\text { LAC7 }-~ \\
\text { Self }\end{array}$ & Mercosur \\
\hline Bolivia & 8.02 & 6.43 & 5.58 & 4.56 & 0.037 & 0.22 & 17.49 & 13.05 \\
Colombia & 10.35 & 5.51 & 0.71 & 3.57 & 0.46 & 0.67 & 4.91 & 1.55 \\
Ecuador & 32.71 & 5.37 & 0.68 & 4.08 & 0.15 & 0.94 & 7.26 & 1.09 \\
Peru & 6.49 & 5.4 & 1.05 & 2.40 & 0.11 & 0.36 & 4.97 & 2.92 \\
Venezuela & 21.06 & 5.9 & 1.5 & 2.5 & 2.7 & 1.03 & 5.3 & 2.3 \\
\hline
\end{tabular}

Source: Direction of Trade Statistics.

counterparts in 1970-86. Moreover, the evidence in the two sub-periods does not establish close association between co-movements in real growth across countries of the Andean group and the corresponding trade linkages with the common sources, as well as other regional blocks in Latin America.

\section{B. The Caribbean}

The majority of Caribbean countries have experienced a reduction in real growth in the latter sample period, compared to the earlier sample period. Table 6 presents summary statistics regarding the mean and standard deviation in Caribbean countries in the two sub-samples: 1970-1986 and 1987-2007.

In the earlier sample period, 1970-1986, co-movements in real GDP growth are

Table 6. Summary Statistics: Real GDP Growth

\begin{tabular}{lcccc}
\hline & \multicolumn{2}{c}{$1987-2006$} & \multicolumn{2}{c}{ 1970-1986 } \\
\hline Caribbean & Mean & Std.Dev. & Mean & Std.Dev. \\
\hline ECCU & 2.3 & 5.1 & 2.7 & 4.2 \\
Caricom & 2.3 & 4.5 & 2.8 & 3.7 \\
The Bahamas & 2.8 & 6.4 & 1.63 & 4.94 \\
Barbados & 2.07 & 2.76 & 3.5 & 6.2 \\
Belize & 1.58 & 3.19 & 4.3 & 4.5 \\
Dominican Republic & 4.83 & 2.42 & 4.1 & 4.3 \\
Guyana & 3.64 & 3.6 & 4.69 & 3.64 \\
Haiti & 3.62 & 0.86 & 3.2 & 3.72 \\
Jamaica & -0.046 & 3.52 & 2.7 & 3.56 \\
Netherlands Antilles & 1.4 & 2.46 & 1.4 & 3.93 \\
Suriname & 3.48 & 3.91 & 6.57 & 5.06 \\
Trinidad \& Tobago & 3.87 & 3.45 & 5.48 & 2.48 \\
\hline
\end{tabular}

Source: World Economic Outlook. 
Table 7a. Co-Movements in Real GDP Growth Sample Period: 1970-1986

\begin{tabular}{|c|c|c|c|c|c|c|c|c|c|c|}
\hline $\begin{array}{l}\text { Caribbean } \\
\text { Group }\end{array}$ & U.S. & EU & Brazil & LAC7 & $\begin{array}{l}\text { ECCU } \\
\text {-Self }\end{array}$ & $\begin{array}{c}\text { Caricom } \\
\text { Self }\end{array}$ & $\begin{array}{c}\text { Caribbean } \\
\text {-Self }\end{array}$ & Andean & & \\
\hline \multirow{2}{*}{ The Bahamas } & 0.17 & $0.45^{* *}$ & 0.15 & 0.19 & -0.014 & -0.11 & -0.07 & -0.14 & -0.20 & 0.23 \\
\hline & $(0.51)$ & $(0.07)$ & $(0.58)$ & $(0.48)$ & $(0.96)$ & $(0.69)$ & $(0.80)$ & $(0.61)$ & $(0.47)$ & $(0.39)$ \\
\hline \multirow[t]{2}{*}{ Barbados } & -0.001 & 0.18 & $0.43 * *$ & $0.49^{*}$ & 0.11 & 0.095 & 0.16 & 0.42 & 0.27 & $0.45^{* *}$ \\
\hline & $(0.99)$ & & & $(0.056)$ & $(0.68)$ & $(0.73)$ & $(0.54)$ & $(0.11)$ & $(0.31)$ & $(0.077)$ \\
\hline \multirow[t]{2}{*}{ Belize } & 0.30 & 0.16 & 0.16 & 0.33 & 0.26 & -0.23 & -0.17 & 0.11 & 0.23 & 0.35 \\
\hline & $(0.24)$ & & & $(0.21)$ & $(0.34)$ & 9) & $.52)$ & $(0.6$ & $(0.40)$ & $(0.18)$ \\
\hline ican & 0.0083 & $30.49 *$ & $0.48^{*}$ & $0.56^{*}$ & -0.15 & $0.43 * *$ & $0.50 *$ & $0.47 * *$ & 0.41 & $0.53 *$ \\
\hline Republic & $(0.97)$ & $(0.05$ & $(0.052)$ & (0.024) & $(0.59)$ & (0.093) & $(0.05)$ & $(0.07)$ & $(0.12)$ & $(0.04)$ \\
\hline \multirow[t]{2}{*}{ ECCU } & $0.57 *$ & $0.48^{*}$ & 0.23 & 0.19 & - & 0.09 - & 0.12 & 0.33 & $0.59 *$ & 0.24 \\
\hline & $(0.02)$ & & $(0.38)$ & $(0.48)$ & & $(0.74)$ & $(0.65)$ & $(0.22)$ & $(0.0$ & $(0.37)$ \\
\hline \multirow[t]{2}{*}{ Guyana } & & & $0.43 * *$ & $0.53 *$ & 0.19 & -0.034 & 0.027 & $0.66^{*}$ & 0.41 & $0.44 * *$ \\
\hline & & & & & & & & & & \\
\hline \multirow[t]{2}{*}{ Haiti } & 0.16 & 0.39 & $0.65^{*}$ & $0.70^{*}$ & 0.088 & 0.40 & $0.50 *$ & 0.40 & 0.25 & 0.65 \\
\hline & $(0.55)$ & $(0.12)$ & $(0.004)$ & $(0.002)$ & $(0.7 .5)$ & $(0.13)$ & $(0.048)$ & $(0.13)$ & $(0.35)$ & $(0.006)$ \\
\hline \multirow[t]{2}{*}{ Jamaica } & 0.02 & 0.21 & -0.30 & -0.24 & 0.12 & $-0.44 * *$ & $-0.47 * *$ & -0.11 & 0.06 & -0.21 \\
\hline & $(0.94)$ & $(0.43)$ & $(0.24)$ & $(0.36)$ & $(0.66)$ & $(0.086)$ & $(0.066)$ & $(0.68)$ & $(0.83)$ & $(0.43)$ \\
\hline Netherland & -0.042 & 0.35 & 0.35 & $0.49 *$ & -0.18 & $0.50^{*}$ & $0.50 *$ & 0.22 & 0.41 & 0.40 \\
\hline Antilles & $(0.87)$ & $(0.17)$ & $(0.17)$ & $(0.05)$ & $(0.51)$ & $(0.05)$ & $(0.05)$ & $(0.42)$ & $(0.11)$ & $(0.12)$ \\
\hline \multirow[t]{2}{*}{ Suriname } & 0.37 & 0.39 & 0.17 & 0.17 & $0.44 * *$ & 0.24 & 0.31 & 0.18 & $0.56^{*}$ & 0.13 \\
\hline & $(0.14)$ & $(0.12)$ & $(0.53)$ & $(0.53)$ & $(0.087)$ & & $(0.23)$ & $(0.51)$ & $(0.023)$ & $(0.63)$ \\
\hline Trinidad \& & -0.31 & 0.33 & 0.36 & $0.47 * *$ & -0.14 & -0.27 & 0.024 & $0.48 *$ & 0.18 & 0.40 \\
\hline Tobago & $(0.23)$ & $(0.19)$ & $(0.16)$ & $(0.06)$ & $(0.60)$ & $(0.31)$ & $(0.93)$ & $(0.05)$ & $(0.50)$ & $(0.13)$ \\
\hline
\end{tabular}

Source: World Economic Outlook.

Magnitudes in parentheses indicate probability of zero correlation.

$*$ and $* *$ denote significance at the five and ten $\%$ levels.

insignificant between Caribbean countries and the U.S. in Table 7a. Co-movements in real growth are significant with real growth in the EU. Trade linkages, in Table $7 \mathrm{~b}$, do not appear to be consistent with significant correlations in real growth across Caribbean countries with the U.S. or the EU, except in a few cases.

Co-movements in real growth in Caribbean countries have shifted over time (see Table 8a). The noticeable increase in trade linkages (see Table $8 \mathrm{~b}$ ) has resulted in significant co-movements between growth in the U.S. and growth in each of The Bahamas, Barbados, Dominican Republic, and the ECCU. Co-movements in real growth with the EU are positive and significant in The Bahamas and Barbados, which appear to be consistent with large trade linkages. 
Table 7b. Average Shares of Trade ((Exports + Imports)/GDP) with Major Trading Partners (in percent) Sample Period: 1970-1986

\begin{tabular}{|c|c|c|c|c|c|c|c|c|c|c|}
\hline $\begin{array}{l}\text { Group of } \\
\text { Caribbean }\end{array}$ & U.S. & EU & Brazil & $\begin{array}{l}\text { ECCU } \\
\text {-Self }\end{array}$ & $\begin{array}{c}\text { Caricom } \\
\text {-Self }\end{array}$ & $\begin{array}{c}\text { Caribbean } \\
\text {-Self }\end{array}$ & Andean & $\begin{array}{l}\text { Central } \\
\text { America }\end{array}$ & LAC7 & Mercosur \\
\hline The Bahamas & 215.4 & 30.3 & 2.5 & 0.02 & 1.4 & 6.3 & 8 & 1.8 & 13.4 & 3.1 \\
\hline Barbados & 26.4 & 22.7 & 0.55 & 0.2 & 12.5 & 13.9 & 3.2 & 0.58 & 4.3 & 1.07 \\
\hline Belize & 48.8 & 33.5 & 0.24 & 0.01 & 4.18 & 7.3 & 1 & 4.4 & 13.7 & 0.3 \\
\hline $\begin{array}{l}\text { Dominican } \\
\text { Republic }\end{array}$ & 15.5 & 3.58 & 0.17 & 0.015 & 0.23 & 0.73 & 2.75 & 0.27 & 3.8 & 0.31 \\
\hline ECCU & 29.7 & 88.9 & 1.8 & 10.2 & 39.9 & 61.3 & 1.7 & 1.8 & 3.1 & 1.8 \\
\hline Guyana & 47.5 & 68.9 & 1.6 & 0.88 & 41.77 & 43.8 & 3.3 & 0.13 & 7 & 2.09 \\
\hline Haiti & 17.02 & 5.79 & 0.17 & 0 & 0.51 & 2.19 & 0.13 & 0.12 & 0.52 & 0.33 \\
\hline Jamaica & 23.7 & 11.9 & 0.33 & 0.23 & 3.6 & 6.8 & 4.7 & 0.47 & 5.4 & 0.45 \\
\hline $\begin{array}{l}\text { Netheriands } \\
\text { Antilles }\end{array}$ & 178.3 & 46.8 & 4.6 & 0.24 & 18.8 & 23.3 & 197.1 & 9.1 & 209.8 & 9.8 \\
\hline Suriname & 25.6 & 25.7 & 1.7 & 0.08 & 7.08 & 8.5 & 1.7 & 0.37 & 3.9 & 2.1 \\
\hline $\begin{array}{l}\text { Trinidad \& } \\
\text { Tobago }\end{array}$ & 42.5 & 13.6 & 0.7 & 0.22 & 6.3 & 7.08 & 2.7 & 1.1 & 2.9 & 0.89 \\
\hline
\end{tabular}

Source: Direction of Trade Statistics.

Table 8a. Co-Movements in Real GDP Growth Sample Period: 1987-2006

\begin{tabular}{|c|c|c|c|c|c|c|c|c|c|c|}
\hline $\begin{array}{l}\text { Group of } \\
\text { Caribbean }\end{array}$ & U.S. & EU & Brazil & LAC7 & $\begin{array}{l}\text { ECCU } \\
\text {-Self }\end{array}$ & $\begin{array}{c}\text { Caricom } \\
\text {-Self }\end{array}$ & $\begin{array}{c}\text { Caribbean- } \\
\text { Self }\end{array}$ & Andean & $\begin{array}{l}\text { Central } \\
\text { America }\end{array}$ & Mercosur \\
\hline The Bahamas & $\begin{array}{c}0.63^{*} \\
(0.003)\end{array}$ & $\begin{array}{l}0.51^{*} \\
(0.02)\end{array}$ & $\begin{array}{c}0.39^{* *} \\
(0.09)\end{array}$ & $\begin{array}{c}0.14 \\
(0.55)\end{array}$ & $\begin{array}{c}0.15 \\
(0.53)\end{array}$ & $\begin{array}{c}0.36 \\
(0.13)\end{array}$ & $\begin{array}{c}0.32 \\
(0.13)\end{array}$ & $\begin{array}{l}-0.06 \\
(0.80)\end{array}$ & $\begin{array}{l}-0.14 \\
(0.60)\end{array}$ & $\begin{array}{c}0.19 \\
(0.43)\end{array}$ \\
\hline Barbados & $\begin{array}{c}0.66^{*} \\
(0.002)\end{array}$ & $\begin{array}{l}0.48^{*} \\
(0.03)\end{array}$ & $\begin{array}{l}0.50^{*} \\
(0.02)\end{array}$ & $\begin{array}{c}0.37 \\
(0.12)\end{array}$ & $\begin{array}{c}0.38 \\
(0.11)\end{array}$ & $\begin{array}{c}0.52 * \\
(0.024)\end{array}$ & $\begin{array}{c}0.52^{*} \\
(0.024)\end{array}$ & $\begin{array}{c}0.06 \\
(0.80)\end{array}$ & $\begin{array}{l}-0.19 \\
(0.44)\end{array}$ & $\begin{array}{l}0.41^{* *} \\
(0.08)\end{array}$ \\
\hline Belize & $\begin{array}{l}-0.08 \\
(0.72)\end{array}$ & $\begin{array}{c}0.17 \\
(0.48)\end{array}$ & $\begin{array}{l}-0.25 \\
(0.28)\end{array}$ & $\begin{array}{c}-0.40 * * \\
(0.09)\end{array}$ & $\begin{array}{c}0.32 \\
(0.18)\end{array}$ & $\begin{array}{l}-0.36 \\
(0.12)\end{array}$ & $\begin{array}{l}-0.36 \\
(0.12)\end{array}$ & $\begin{array}{l}-0.57^{*} \\
(0.011)\end{array}$ & $\begin{array}{l}-0.22 \\
(0.36)\end{array}$ & $\begin{array}{l}0.42 * \\
(0.07)\end{array}$ \\
\hline $\begin{array}{l}\text { Dominican } \\
\text { Republic }\end{array}$ & $\begin{array}{c}0.49^{* *} \\
(0.03)\end{array}$ & $\begin{array}{c}0.17 \\
(0.48)\end{array}$ & $\begin{array}{c}0.42 * * \\
(0.07)\end{array}$ & $\begin{array}{c}0.37 \\
(0.12)\end{array}$ & $\begin{array}{l}-0.06 \\
(0.82)\end{array}$ & $\begin{array}{c}0.15 \\
(0.53)\end{array}$ & $\begin{array}{c}0.12 \\
(0.63)\end{array}$ & $\begin{array}{l}0.041 \\
(0.87)\end{array}$ & $\begin{array}{c}0.13 \\
(0.59)\end{array}$ & $\begin{array}{c}0.38 \\
(0.11)\end{array}$ \\
\hline ECCU & $\begin{array}{c}0.60^{*} \\
(0.007)\end{array}$ & $\begin{array}{c}0.47 \\
(0.045)\end{array}$ & $\begin{array}{l}-0.23 \\
(0.35)\end{array}$ & $\begin{array}{l}-0.10 \\
(0.69)\end{array}$ & $\begin{array}{l}-0.27 \\
(0.27)\end{array}$ & $\begin{array}{c}0.19 \\
(0.44)\end{array}$ & $\begin{array}{l}-0.11 \\
(0.66)\end{array}$ & $\begin{array}{l}-0.27 \\
(0.27)\end{array}$ & $\begin{array}{l}-0.33 \\
(0.17)\end{array}$ & $\begin{array}{l}-0.13 \\
(06.0)\end{array}$ \\
\hline Guyana & $\begin{array}{l}-0.05 \\
(0.83)\end{array}$ & $\begin{array}{l}-0.50^{*} \\
(0.025)\end{array}$ & $\begin{array}{l}0.36^{*} \\
(0.12)\end{array}$ & $\begin{array}{c}0.51^{*} \\
(0.024)\end{array}$ & $\begin{array}{c}-0.42 * * \\
(0.08)\end{array}$ & $\begin{array}{l}-0.22 \\
(0.37)\end{array}$ & $\begin{array}{l}-0.19 \\
(0.42)\end{array}$ & $\begin{array}{l}0.46^{*} \\
(0.05)\end{array}$ & $\begin{array}{l}0.43 * * \\
(0.07)\end{array}$ & $\begin{array}{l}0.52 * \\
(0.02)\end{array}$ \\
\hline Haiti & $\begin{array}{l}-0.01 \\
(0.97)\end{array}$ & $\begin{array}{c}0.26 \\
(0.27)\end{array}$ & $\begin{array}{l}-0.28 \\
(0.23)\end{array}$ & $\begin{array}{l}-0.27 \\
(0.27)\end{array}$ & $\begin{array}{c}0.08 \\
(0.75)\end{array}$ & $\begin{array}{c}0.32 \\
(0.18)\end{array}$ & $\begin{array}{c}0.23 \\
(0.34)\end{array}$ & $\begin{array}{l}-0.15 \\
(0.55)\end{array}$ & $\begin{array}{l}0.44 * * \\
(0.06)\end{array}$ & $\begin{array}{l}-0.15 \\
(0.54)\end{array}$ \\
\hline Jamaica & $\begin{array}{l}-0.25 \\
(0.29)\end{array}$ & $\begin{array}{l}-0.11 \\
(0.66)\end{array}$ & $\begin{array}{l}-0.016 \\
(0.95)\end{array}$ & $\begin{array}{l}-0.14 \\
(0.58)\end{array}$ & $\begin{array}{l}-0.17 \\
(0.48)\end{array}$ & $\begin{array}{l}-0.053 \\
(0.83)\end{array}$ & $\begin{array}{l}0.025 \\
(0.92)\end{array}$ & $\begin{array}{l}-0.13 \\
(0.61)\end{array}$ & $\begin{array}{l}0.44 * * \\
(0.06)\end{array}$ & $\begin{array}{l}-0.15 \\
(0.54)\end{array}$ \\
\hline $\begin{array}{l}\text { Netheriands } \\
\text { Antilles }\end{array}$ & $\begin{array}{l}-0.10 \\
(0.67)\end{array}$ & $\begin{array}{l}-0.19 \\
(0.42)\end{array}$ & $\begin{array}{c}0.27 \\
(0.25)\end{array}$ & $\begin{array}{c}0.20 \\
(0.41)\end{array}$ & $\begin{array}{c}0.08 \\
(0.75)\end{array}$ & $\begin{array}{c}-0.42 * * \\
(0.08)\end{array}$ & $\begin{array}{c}-0.42 * * \\
(0.07)\end{array}$ & $\begin{array}{l}-0.076 \\
(0.75)\end{array}$ & $\begin{array}{l}-0.04 \\
(0.86)\end{array}$ & $\begin{array}{c}0.30 \\
(0.21)\end{array}$ \\
\hline Suriname & $\begin{array}{l}0.081 \\
(0.73)\end{array}$ & $\begin{array}{c}0.28 \\
(0.23)\end{array}$ & $\begin{array}{l}-0.054 \\
(0.82)\end{array}$ & $\begin{array}{c}0.05 \\
(0.84)\end{array}$ & $\begin{array}{c}0.29 \\
(0.23)\end{array}$ & $\begin{array}{c}0.22 \\
(0.37)\end{array}$ & $\begin{array}{c}0.22 \\
(0.37)\end{array}$ & $\begin{array}{c}0.16 \\
(0.52)\end{array}$ & $\begin{array}{l}-0.54^{*} \\
(0.017)\end{array}$ & $\begin{array}{c}0.06 \\
(0.81)\end{array}$ \\
\hline $\begin{array}{l}\text { Trinidad \& } \\
\text { Tobago }\end{array}$ & $\begin{array}{c}0.08 \\
(0.74)\end{array}$ & $\begin{array}{l}-0.022 \\
(0.93)\end{array}$ & $\begin{array}{c}0.09 \\
(0.71)\end{array}$ & $\begin{array}{c}0.24 \\
(0.32)\end{array}$ & $\begin{array}{l}-0.21 \\
(0.39)\end{array}$ & $\begin{array}{c}0.46^{*} \\
(0.046)\end{array}$ & $\begin{array}{c}0.19 \\
(0.44)\end{array}$ & $\begin{array}{c}0.29 \\
(0.23)\end{array}$ & $\begin{array}{c}0.33 \\
(0.17)\end{array}$ & $\begin{array}{c}0.17 \\
(0.47)\end{array}$ \\
\hline
\end{tabular}

Source: World Economic Outlook.

Magnitudes in parentheses indicate probability of zero correlation.

$*$ and $* *$ denote significance at the five and ten percent levels. 
Table 8b. Average Shares of Trade ((Exports + Imports)/GDP) with Major Trading Partners (in percent) Sample Period: 1987-2006

\begin{tabular}{lcccccccccc}
\hline $\begin{array}{l}\text { Group of } \\
\text { Caribbean }\end{array}$ & U.S. & EU & Brazil & $\begin{array}{c}\text { ECCU } \\
\text {-Self }\end{array}$ & $\begin{array}{c}\text { Caricom } \\
\text {-Self }\end{array}$ & $\begin{array}{c}\text { Caribbean } \\
\text {-Self }\end{array}$ & Andean & $\begin{array}{c}\text { Central } \\
\text { America }\end{array}$ & LAC7 & Mercosur \\
\hline $\begin{array}{l}\text { The } \\
\text { Bahamas }\end{array}$ & 46.5 & 27.0 & 2.98 & 0.009 & 0.48 & 2.08 & 1.9 & 0.49 & 6.56 & 3.4 \\
Barbados & 17.9 & 9.04 & 0.59 & 2.0 & 11.1 & 11.9 & 1.19 & 0.48 & 2.15 & 0.72 \\
Belize & 36.4 & 16.5 & 0.28 & 0.07 & 3.68 & 5.3 & 0.53 & 3.34 & 7.6 & 0.42 \\
$\begin{array}{l}\text { Dominican } \\
\text { Republic }\end{array}$ & 32.71 & 5.37 & 0.68 & 0.016 & 0.64 & 0.94 & 4.08 & 0.94 & 7.26 & 1.096 \\
ECCU & 141.9 & 115.9 & 7.9 & 18.3 & 86.7 & 89.0 & 4.3 & 2.2 & 13.8 & 8.8 \\
Guyana & 42.4 & 44.3 & 1.34 & 1.7 & 22.5 & 35.6 & 1.8 & 0.23 & 9.3 & 7.2 \\
Haiti & 17.8 & 3.53 & 0.4 & 0.004 & 0.34 & 2.83 & 1.09 & 0.68 & 2.07 & 0.71 \\
Jamaica & 29.1 & 10.97 & 0.97 & 0.36 & 4.85 & 5.49 & 2.01 & 0.73 & 4.4 & 1.13 \\
Netheriands & 35.7 & 20.5 & 2.8 & 0.48 & 11.7 & 13.6 & 61.8 & 9.5 & 69.8 & 4.7 \\
Antilles & & & & & & & & & & \\
Suriname & 43.8 & 46.4 & 7.2 & 0.05 & 14.4 & 18.5 & 3.2 & 0.73 & 10.7 & 4.7 \\
Trinidad \& & 34.8 & 9.7 & 1.8 & 1.51 & 8.8 & 10.03 & 5.4 & 1.1 & 8.3 & 2.2 \\
Tobago & & & & & & & & & & \\
\hline
\end{tabular}

Source: Direction of Trade Statistics.

Table 9. Summary Statistics: Real GDP Growth

\begin{tabular}{|c|c|c|c|c|}
\hline \multirow{2}{*}{ Central America } & \multicolumn{2}{|c|}{$1987-2006$} & \multicolumn{2}{|c|}{ 1970-1986 } \\
\hline & Mean & Std. Dev. & Mean & Std. Dev. \\
\hline Costa Rica & 4.8 & 2.4 & 4.3 & 4.29 \\
\hline El Salvador & 3.50 & 1.96 & 1.64 & 4.76 \\
\hline Guatemala & 3.62 & 0.86 & 3.35 & 3.66 \\
\hline Honduras & 3.52 & 2.25 & 3.65 & 4.04 \\
\hline Nicaragua & 1.99 & 4.26 & 0.96 & 8.72 \\
\hline Panama & 3.87 & 5.04 & 4.33 & 3.53 \\
\hline
\end{tabular}

Source: World Economic Outlook.

\section{Central America}

In general, real growth has picked up over time, although with varying rates, across countries of Latin America. Table 9. presents summary statistics of real growth across countries of Central America. Except for Panama, variability of real growth appears pronouncedly smaller in the latter period, 1987-2007, compared to the earlier period, 1970-1986.

Consistent with strong trade linkages, real growth in Costa Rica and Guatemala varies significantly with growth in the U.S. Tables $10 \mathrm{a}, \mathrm{b}$ present co-movements in real growth and trade linkages across countries of Central America in the period 
Table 10a. Co-Movements in Real GDP Growth Sample Period: 1970-1986

\begin{tabular}{lcccccccc}
\hline $\begin{array}{l}\text { Group of } \\
\text { Central America }\end{array}$ & U.S. & EU & Brazil & $\begin{array}{c}\text { Andean } \\
\text {-Self }\end{array}$ & Caribbean & $\begin{array}{c}\text { Central } \\
\text { America }\end{array}$ & $\begin{array}{c}\text { LAC7 } \\
\text {-Self }\end{array}$ & $\begin{array}{c}\text { Mercosur } \\
\text {-Self }\end{array}$ \\
\hline Costa Rica & $0.61^{*}$ & $0.72^{*}$ & $0.48^{*}$ & 0.36 & 0.25 & $0.45^{* *}$ & $0.61^{*}$ & $0.64^{*}$ \\
& $(0.0097)$ & $(0.001)$ & $(0.18)$ & $(0.18)$ & $(0.035)$ & $(0.078)$ & $(0.013)$ & $(0.007)$ \\
El Salvador & 0.40 & $0.57^{*}$ & 0.39 & 0.27 & 0.14 & 0.20 & 0.36 & 0.40 \\
& $(0.11)$ & $(0.017)$ & $(0.12)$ & $(0.32)$ & $(0.61)$ & $(0.45)$ & $(0.17)$ & $(0.13)$ \\
Guatemala & 0.23 & $0.65^{*}$ & $0.63^{*}$ & $0.59^{*}$ & $0.74^{*}$ & $0.63^{*}$ & $0.76^{*}$ & $0.69^{*}$ \\
& $(0.37)$ & $(0.005)$ & $(0.007)$ & $(0.016)$ & $(0.001)$ & $(0.009)$ & $(0.0006)$ & $(0.003)$ \\
Honduras & $0.62^{*}$ & $0.66^{*}$ & 0.26 & 0.15 & $0.53^{*}$ & 0.34 & 0.35 & 0.33 \\
& $(0.008)$ & $(0.004)$ & $(0.32)$ & $(0.58)$ & $(0.035)$ & $(0.19)$ & $(0.19)$ & $(0.22)$ \\
Nicaragua & -0.12 & -0.05 & 0.071 & 0.21 & -0.11 & 0.067 & 0.012 & 0.022 \\
& $(0.66)$ & $(0.86)$ & $(0.79)$ & $(0.44)$ & $(0.67)$ & $(0.80)$ & $(0.96)$ & $(0.93)$ \\
Panama & -0.083 & 0.099 & 0.17 & 0.34 & 0.21 & -0.20 & 0.27 & 0.16 \\
& $(0.75)$ & $(0.71)$ & $(0.52)$ & $(0.20)$ & $(0.44)$ & $(0.46)$ & $(0.32)$ & $(0.56)$ \\
\hline
\end{tabular}

Source: World Economic Outlook.

Magnitudes in parentheses indicate probability of zero correlation.

*and ** denote significance at the five and ten percent levels.

1970-1986. Growth in other Central American countries does not vary significantly with the U.S., despite strong trade linkages.

Significant co-movements in real growth in Central America with growth in the EU are more pervasive. While trade linkages may explain this evidence, in part, co-movements in real growth in Nicaragua and Panama are not significant with real growth in the EU, despite large shares of trade.

Real growth in Brazil was a major determinant of growth in Costa Rica and Guatemala in 1970-86. Nonetheless, the shares of trade with Brazil appear, in general, limited across countries of Central America.

Compared to the earlier period, significant co-movements in real growth appear

Table 10b. Average Shares of Trade ((Exports + Imports)/GDP) with Major Trading Partners (in percent) Sample Period: 1970-1986

\begin{tabular}{lcccccccc}
\hline $\begin{array}{l}\text { Group of } \\
\begin{array}{l}\text { Central } \\
\text { America }\end{array}\end{array}$ & U.S. & EU & Brazil & $\begin{array}{c}\text { Andean } \\
\text {-Self }\end{array}$ & Caribbean & $\begin{array}{c}\text { Central } \\
\text { America }\end{array}$ & $\begin{array}{c}\text { LAC7 } \\
\text {-Self }\end{array}$ & $\begin{array}{c}\text { Mercosur } \\
\text {-Self }\end{array}$ \\
\hline Costa Rica & 20.60 & 13.11 & 0.39 & 2.71 & 1.13 & 11.83 & 4.84 & 0.64 \\
El Salvador & 16.32 & 11.37 & 0.15 & 2.43 & 0.14 & 13.85 & 4.02 & 0.25 \\
Guatemala & 10.54 & 6.96 & 0.14 & 1.43 & 0.83 & 7.12 & 2.58 & 0.21 \\
Honduras & 25.52 & 9.91 & 0.41 & 2.75 & 2.51 & 6.22 & 4.06 & 0.53 \\
Nicaragua & 18.73 & 14.06 & 0.37 & 3.65 & 0.59 & 15.3 & 6.81 & 0.77 \\
Panama & 15.68 & 5.33 & 0.31 & 6.73 & 0.64 & 2.12 & 5.35 & 0.49 \\
\hline
\end{tabular}

Source: Direction of Trade Statistics. 
Table 11a. Co-Movements in Real GDP Growth Sample Period: 1987-2006

\begin{tabular}{lcccccccc}
\hline $\begin{array}{l}\text { Group of } \\
\text { Central America }\end{array}$ & U.S. & EU & Brazil & \multicolumn{1}{c}{ Andean Caribbean $\begin{array}{c}\text { Central } \\
\text { America-Self }\end{array}$} & LAC7 & Mercosur \\
\hline Costa Rica & $0.44^{*}$ & -0.15 & -0.097 & -0.13 & 0.16 & 0.32 & 0.06 & 0.064 \\
& $(0.049)$ & $(0.52)$ & $(0.68)$ & $(0.60)$ & $(0.52)$ & $(0.19)$ & $(0.80)$ & $(0.80)$ \\
El Salvador & -0.0086 & -0.41 & 0.013 & 0.36 & -0.36 & 0.32 & 0.20 & 0.19 \\
& $(0.97)$ & $(0.07)$ & $(0.96)$ & $(0.14)$ & $(0.14)$ & $(0.19)$ & $(0.42)$ & $(0.43)$ \\
Guatemala & $0.42^{* *}$ & 0.26 & 0.11 & 0.0087 & -0.28 & 0.19 & 0.19 & 0.22 \\
& $(0.07)$ & $(0.27)$ & $(0.65)$ & $(0.97)$ & $(0.24)$ & $(0.44)$ & $(0.44)$ & $(0.38)$ \\
Honduras & 0.034 & -0.15 & 0.30 & 0.25 & -0.082 & -0.06 & 0.37 & $0.42^{* *}$ \\
& $(0.89)$ & $(0.53)$ & $(0.20)$ & $(0.30)$ & $(0.74)$ & $(0.81)$ & $(0.12)$ & $(0.075)$ \\
Nicaragua & 0.11 & -0.097 & 0.32 & 0.34 & $0.59^{*}$ & $0.63 *$ & $0.40 *$ & 0.35 \\
& $(0.64)$ & $(0.68)$ & $(0.17)$ & $(0.15)$ & $(0.0083)$ & $(0.0042)$ & $(0.09)$ & $(0.15)$ \\
Panama & -0.17 & $-0.47^{*}$ & -0.06 & 0.34 & 0.33 & $0.54 *$ & 0.35 & 0.21 \\
& $(0.48)$ & $(0.036)$ & $(0.79)$ & $(0.16)$ & $(0.17)$ & $(0.017)$ & $(0.15)$ & $(0.39)$ \\
\hline
\end{tabular}

Source: World Economic Outlook.

Magnitudes in parenthesis indicate probability of zero correlation.

$*$ and ${ }^{* *}$ denote significance at the five and ten percent levels.

Table 11b. Average Shares of Trade ((Exports + Imports)/GDP) with Major Trading Partners (in percent) Sample Period: 1987-2006

\begin{tabular}{lcccccccc}
\hline $\begin{array}{l}\text { Group of } \\
\text { Central America }\end{array}$ & U.S. & EU & Brazil & Andean & $\begin{array}{c}\text { Carib } \\
\text { bean }\end{array}$ & $\begin{array}{c}\text { Central } \\
\text { America-Self }\end{array}$ & LAC7 & Mercosur \\
\hline Costa Rica & 24.0 & 10.2 & 1.05 & 3.17 & 0.74 & 6.82 & 6.88 & 1.67 \\
El Salvador & 21.5 & 5.3 & 0.49 & 2.05 & 0.51 & 11.32 & 4.77 & 0.81 \\
Guatemala & 16.88 & 4.5 & 0.5 & 1.67 & 1.03 & 7.23 & 5.02 & 0.9 \\
Honduras & 35.06 & 8.67 & 0.73 & 1.56 & 1.34 & 9.92 & 4.85 & 1.08 \\
Nicaragua & 14.27 & 10.4 & 0.15 & 5.43 & 0.15 & 14.85 & 7.81 & 0.69 \\
Panama & 12.74 & 4.52 & 0.48 & 3.65 & 0.95 & 2.78 & 4.29 & 0.88 \\
\hline
\end{tabular}

Source: Direction of Trade Statistics.

less pervasive between countries of Central America and the common sources. Tables $11 \mathrm{a}, \mathrm{b}$ present co-movements in real growth and trade linkages across countries in Central America in the period 1987-2007. Despite large trade linkages, co-movements in real growth with the U.S. and the EU are negative in a few countries.

\section{LAC7 group}

In general, average real growth is lower and variability is less pronounced in the latter period, compared to the earlier period. Table 12 summarizes statistics characterizing real growth across countries of LAC7 in 1970-1986 and 1987-2007. 
Table 12. Summary Statistics: Real GDP Growth

\begin{tabular}{lcccc}
\hline \multirow{2}{*}{ LAC7 Group } & \multicolumn{2}{c}{$1987-2006$} & \multicolumn{2}{c}{$1970-1986$} \\
\cline { 2 - 5 } & Mean & Std. Dev. & Mean & Std. Dev. \\
\hline Argentina & 2.8 & 6.4 & 1.53 & 4.8 \\
Brazil & 2.2 & 2.4 & 6.01 & 5.06 \\
Chile & 5.9 & 3.1 & 1.96 & 7.2 \\
Colombia & 3.3 & 2.3 & 4.82 & 2.4 \\
Mexico & 3.04 & 2.82 & 4.87 & 4.2 \\
Peru & 2.4 & 6.2 & 3.2 & 4.9 \\
Venezuela & 2.5 & 6.84 & 3.09 & 3.9 \\
\hline
\end{tabular}

Source: World Economic Outlook.

Table 13a. Co-Movements in Real GDP Growth Sample Period: 1970-1986

\begin{tabular}{lcccccccc}
\hline $\begin{array}{l}\text { Group of } \\
\text { LAC7 }\end{array}$ & U.S. & EU & Brazil & $\begin{array}{c}\text { Andean } \\
\text {-Self }\end{array}$ & Caribbean & $\begin{array}{c}\text { Central } \\
\text { America }\end{array}$ & $\begin{array}{c}\text { LAC7 }-\begin{array}{c}\text { Mercosur- } \\
\text { Self }\end{array} \\
\text { Self }\end{array}$ \\
\hline Argentina & 0.24 & $0.61^{*}$ & 0.14 & 0.21 & -0.05 & 0.28 & $0.61^{*}$ & $0.71^{*}$ \\
& $(0.38)$ & $(0.013)$ & $(0.60)$ & $(0.44)$ & $(0.86)$ & $(0.31)$ & $(0.016)$ & $(0.0032)$ \\
Brazil & $0.62^{*}$ & $0.46^{*}$ & & 0.0022 & $0.67^{*}$ & $0.76^{*}$ & $0.47^{* *}$ & 0.16 \\
& $(0.01)$ & $(0.07)$ & & $(0.99)$ & $(0.006)$ & $(0.009)$ & $(0.075)$ & $(0.56)$ \\
Chile & 0.19 & $0.68^{*}$ & $0.61^{*}$ & 0.13 & $0.52^{*}$ & $0.78^{*}$ & 0.35 & 0.28 \\
& $(0.48)$ & $(0.004)$ & $(0.012)$ & $(0.65)$ & $(0.047)$ & $(0.0007)$ & $(0.20)$ & $(0.31)$ \\
Colombia & 0.35 & $0.67^{*}$ & $0.52^{*}$ & $0.49^{* *}$ & $0.43^{* *}$ & $0.64^{*}$ & $0.96^{*}$ & $0.84^{*}$ \\
& $(0.19)$ & $(0.004)$ & $(0.04)$ & $(0.06)$ & $(0.10)$ & $(0.0098)$ & $(0.0001)$ & $(0.0001)$ \\
Mexico & $0.64^{*}$ & $0.66^{*}$ & $0.35^{*}$ & $0.62^{*}$ & 0.27 & $0.49^{* *}$ & 0.57 & $0.72^{*}$ \\
& $(0.0078)$ & $(0.006)$ & $(0.18)$ & $(0.014)$ & $(0.34)$ & $(0.067)$ & $(0.025)$ & $(0.0027)$ \\
Peru & 0.18 & 0.036 & 0.13 & 0.20 & $0.58^{*}$ & 0.31 & -0.03 & -0.032 \\
& $(0.49)$ & $(0.80)$ & $(0.63)$ & $(0.48)$ & $(0.023)$ & $(0.27)$ & $(0.91)$ & $(0.091)$ \\
Venezuela & 0.42 & $0.67^{*}$ & 0.13 & $0.44^{* *}$ & -0.03 & 0.36 & $0.76^{*}$ & $0.99^{*}$ \\
& $(0.10)$ & $(0.005)$ & $(0.63)$ & $(0.097)$ & $(0.90)$ & $(0.19)$ & $(0.001)$ & $(0.001)$ \\
\hline
\end{tabular}

Source: World Economic Outlook.

Magnitudes in parentheses indicate probability of zero correlation.

$*$ and ** denote significance at the five and ten percent levels.

Despite large trade shares with the U.S, co-movements in real growth in LAC7 are generally larger with the EU, compared to the U.S. Tables $13 \mathrm{a}, \mathrm{b}$ present comovements in real growth and trade linkages across LAC7 in the period 19701986. In general, significant co-movements in real growth do not appear to be readily explained by large trade linkages with countries in LAC7.

Significant co-movements in real growth are less pervasive in 1987-2006. Tables $14 \mathrm{a}, \mathrm{b}$ present co-movements in real growth and trade linkages across countries of LAC7 in the period 1987-2007. Despite large trade shares with the 
Table 13b. Average Shares of Trade ((Exports + Imports)/GDP) with Major Trading Partners (in percent) Sample Period: 1970-1986

\begin{tabular}{lcccccccc}
\hline Group LAC7 & U.S. & EU & Brazil & $\begin{array}{c}\text { Andean } \\
\text {-Self }\end{array}$ & Caribbean & $\begin{array}{c}\text { Centrai } \\
\text { America }\end{array}$ & $\begin{array}{c}\text { LAC7 } \\
\text {-Self }\end{array}$ & $\begin{array}{c}\text { Mercosur } \\
\text {-Self }\end{array}$ \\
\hline Argentina & 1.50 & 3.68 & 0.99 & 0.63 & 0.048 & 0.047 & 1.85 & 1.63 \\
Brazil & 5.26 & 7.16 & & 0.77 & 0.14 & 0.093 & 2.22 & 1.63 \\
Chile & 6.57 & 10.53 & 2.24 & 2.47 & 0.16 & 0.095 & 6.45 & 4.63 \\
Colombia & 10.35 & 5.51 & 0.71 & 3.57 & 0.46 & 0.67 & 4.91 & 1.55 \\
Mexico & 8.47 & 2.35 & 0.28 & 0.18 & 0.10 & 0.20 & 0.60 & 0.46 \\
Peru & 7.85 & 6.25 & 0.74 & 1.53 & 0.13 & 0.20 & 2.9 & 1.79 \\
Venezuela & 15.24 & 7.4 & 0.89 & 0.66 & 3.95 & 0.86 & 2.3 & 1.5 \\
\hline
\end{tabular}

Source: Direction of Trade Statistics.

U.S., co-movements in real growth with the U.S. are not significant. Significant comovements in real growth with other countries in LAC7 and other regional blocks in Latin America do not reflect relatively larger trade linkages, in general.

There is a significant co-movement in stock price indices in Argentina and Brazil with the U.S. and the EU. Table 15. presents co-movements in stock price indices between the group of LAC7 and the common sources in 1987-2007. ${ }^{17}$ Further, stock price indices in Argentina, Chile, Colombia, Mexico, Peru, and

Table 14a. Co-Movements in Real GDP Growth Sample Period: 1987-2006

\begin{tabular}{lcccccccc}
\hline LAC7 Group & U.S. & EU & Brazil & $\begin{array}{c}\text { Andean } \\
\text {-Self }\end{array}$ & Caribbean & $\begin{array}{c}\text { Central } \\
\text { America }\end{array}$ & $\begin{array}{c}\text { LAC7 } \\
\text {-Self }\end{array}$ & $\begin{array}{c}\text { Mercosur } \\
\text {-Self }\end{array}$ \\
\hline \multirow{2}{*}{ Argentina } & 0.12 & -0.38 & 0.12 & $0.62^{*}$ & 0.16 & $0.53^{*}$ & $0.77^{*}$ & 0.18 \\
& $(0.61)$ & $(0.10)$ & $(0.60)$ & $(0.004)$ & $(0.53)$ & $(0.019)$ & $(0.0001)$ & $(0.46)$ \\
Brazil & 0.28 & 0.05 & & $0.40^{* *}$ & 0.25 & 0.04 & 0.21 & 0.18 \\
& $(0.23)$ & $(0.84)$ & & $(0.087)$ & $(0.31)$ & $(0.87)$ & $(0.38)$ & $(0.47)$ \\
Chile & -0.005 & -0.096 & 0.21 & 0.17 & $-0.43^{* *}$ & 0.044 & 0.23 & 0.31 \\
& $(0.98)$ & $(0.69)$ & $(0.38)$ & $(0.48)$ & $(0.07)$ & $(0.86)$ & $(0.35)$ & $(0.20)$ \\
Colombia & -0.05 & -0.16 & 0.32 & $0.42^{* *}$ & -0.09 & 0.034 & 0.35 & $0.47^{*}$ \\
& $(0.82)$ & $(05.0)$ & $(0.17)$ & $(0.07)$ & $(0.71)$ & $(0.89)$ & $(0.14)$ & $(0.04)$ \\
Mexico & 0.35 & 0.15 & -0.09 & -0.074 & 0.13 & 0.28 & 0.17 & 0.064 \\
& $(0.13)$ & $(0.52)$ & $(0.71)$ & $(0.76)$ & $(0.59)$ & $(0.24)$ & $(0.50)$ & $(0.80)$ \\
Peru & 0.014 & -0.30 & $0.56^{*}$ & 0.19 & $0.39^{* *}$ & $0.47^{*}$ & 0.25 & $0.60^{*}$ \\
& $(0.95)$ & $(0.19)$ & $(0.01)$ & $(0.45)$ & $(0.097)$ & $(0.04)$ & $(0.31)$ & $(0.006)$ \\
Venezuela & -0.04 & 0.017 & 0.034 & 0.36 & -0.12 & 0.16 & $0.50^{*}$ & 0.28 \\
& $(0.86)$ & $(0.94)$ & $(0.89)$ & $(0.13)$ & $(0.62)$ & $(0.51)$ & $(0.029)$ & $(0.25)$ \\
\hline
\end{tabular}

Source: World Economic Outlook.

Magnitudes in parentheses indicate the probability of zero correlation.

$*$ and $* *$ denote significance at the five and ten percent levels.

\footnotetext{
${ }^{17}$ Data are not available to illustrate stock price co-movements for other countries in Latin America.
} 
Table 14b. Average Shares of Trade((Exports + Imports)/GDP) with Major Trading Partners (in percent) Sample Period: 1987-2006

\begin{tabular}{lcccccccc}
\hline LAC7 Group & U.S. & EU & Brazil & $\begin{array}{c}\text { Andean } \\
\text {-Self }\end{array}$ & $\begin{array}{c}\text { Carib } \\
\text {-bean }\end{array}$ & $\begin{array}{c}\text { Centrai } \\
\text { America }\end{array}$ & $\begin{array}{c}\text { LAC7 } \\
\text {-Self }\end{array}$ & $\begin{array}{c}\text { Mercosur } \\
\text {-Self }\end{array}$ \\
\hline Argentina & 2.76 & 4.54 & 4.18 & 0.73 & 0.067 & 0.082 & 6.2 & 6.05 \\
Brazil & 3.9 & 4.85 & & 0.60 & 0.12 & 0.09 & 2.69 & 2.32 \\
Chile & 8.76 & 11.77 & & 2.71 & 0.21 & 0.24 & 9.86 & 6.99 \\
Colombia & 6.88 & 6.43 & 0.32 & 1.63 & 0.34 & 0.37 & 2.33 & 0.90 \\
Mexico & 34.17 & 3.65 & 0.44 & 0.42 & 0.19 & 0.36 & 1.17 & 0.85 \\
Peru & 6.49 & 5.4 & 1.05 & 2.4 & 0.11 & 0.36 & 4.97 & 2.92 \\
Venezuela & 21.06 & 5.9 & 1.5 & 2.5 & 2.7 & 1.03 & 5.3 & 2.3 \\
\hline
\end{tabular}

Source: Direction of Trade Statistics.

Venezuela move significantly with Brazil. Significant co-movements in stock price indices do not correspond to significant co-movements in real output growth.

\section{E. Mercosur group}

Except for Brazil and Paraguay, average real growth is higher in 1987-2007, compared to 1970-1986. Table 16 summarizes major statistics, describing the average and variability of real growth across Mercosur countries.

Despite large trade shares with the EU, Brazil and other Mercosur countries, comovements with real growth in Paraguay and Uruguay are not significant. Table $17 \mathrm{a}, \mathrm{b}$ presents co-movements in real growth and trade linkages across Mercosur countries in the period 1970-1986.

Significant co-movement between real growth in Uruguay with respect to growth in the U.S. and LAC7 corresponds to high trade shares. Tables $18 \mathrm{a}, \mathrm{b}$ present co-movements in real growth and trade linkages across Mercosur countries in the period 1987-2007.

Overall, the evidence across the various regional blocks of Latin America indicates strong co-movements in real growth with growth in the common sources and other regional blocks in the earlier sample period, 1970-1986, compared to the latter period, 1987-2007. While regional integration was presumably lower in the earlier sample period, the higher correlation coefficient could be capturing the impact of a change in a third element that impacted all countries within that period. More specifically, all countries in the $1970-86$ periods were buffeted by the effects of the two oil crises, causing incomes to move together and in the same direction. Moreover, trade linkages do not appear to be closely related to significant comovements in real growth across sources and regional blocks over time. 
Table 15. Co-Movements In-Stock Price Indices Sample Period: 1987-2006

\begin{tabular}{lccc}
\hline Group of LAC7 & U.S. & EU & Brazil \\
\hline Argentina & $0.58^{*}$ & $0.58^{*}$ & $0.72^{*}$ \\
& $(0.009)$ & $(0.009)$ & $(0.0005)$ \\
Brazil & $0.41^{* *}$ & $0.46^{*}$ & \\
& $(0.081)$ & $(0.05)$ & \\
Chile & 0.32 & 0.33 & $0.69^{*}$ \\
& $(0.19)$ & $(0.17)$ & $(0.001)$ \\
Colombia & -0.12 & 0.022 & $0.44^{* *}$ \\
Mexico & $(0.69)$ & $(0.94)$ & $(0.06)$ \\
& 0.29 & 0.29 & $0.44^{* *}$ \\
Peru & $(0.22)$ & $(0.23)$ & $(0.06)$ \\
& 0.017 & 0.1 & $0.64^{*}$ \\
Venezuela & $(0.95)$ & $(0.73)$ & $(0.013)$ \\
& 0.16 & 0.32 & $0.57^{*}$ \\
& $(0.59)$ & $(0.27)$ & $(0.03)$ \\
\hline
\end{tabular}

Source: World Economic Outlook.

Bracketed magnitudes indicate the probability of zero correlation.

* and ${ }^{* *}$ denote significance at the five and ten percen levels.

Table 16. Summary Statistics: Real GDP Growth

\begin{tabular}{ccccc}
\hline \multirow{2}{*}{ Mercosur } & \multicolumn{2}{c}{$1987-2006$} & \multicolumn{2}{c}{$1970-1986$} \\
\cline { 2 - 5 } & Mean & Std. Dev. & Mean & Std. Dev. \\
\hline Argentina & 4.18 & 2.96 & 5.53 & 4.15 \\
Brazil & 3.54 & 1.33 & 1.97 & 3.77 \\
Chile & 2.21 & 2.37 & 6.22 & 5.14 \\
Paraguay & 1.99 & 4.26 & 0.94 & 9.00 \\
Uruguay & 4.6 & 5.19 & 0.97 & 5.29 \\
\hline
\end{tabular}

Source: World Economic Outlook.

\section{The Impact of Trade and Financial Linkages on Co-Movements in Real Output Growth}

\section{A. Basic results}

To set the stage, time-series regressions analyze developments in real growth within countries with the change in trade shares over time. Trade shares are measured with respect to the common sources and regional blocks. ${ }^{18}$

The evidence illustrates the influence of trade relations with specific trading

${ }^{18}$ Detailed results are available upon request. 
Table 17a. Co-Movements in Real GDP Growth Sample Period: 1970-1986

\begin{tabular}{lcccccccc}
\hline $\begin{array}{l}\text { Mercosur } \\
\text { Group }\end{array}$ & U.S. & EU & Brazil & $\begin{array}{c}\text { Andean } \\
\text {-Self }\end{array}$ & $\begin{array}{c}\text { Caribbean } \\
\text { Central } \\
\text { America }\end{array}$ & $\begin{array}{c}\text { LAC7 } \\
\text {-Self }\end{array}$ & $\begin{array}{c}\text { Mercosur } \\
\text {-Self }\end{array}$ \\
\hline Argentina & 0.16 & $0.41^{* *}$ & $0.44^{* *}$ & 0.33 & 0.39 & 0.17 & 0.14 & $0.43^{* *}$ \\
& $(0.53)$ & $(0.10)$ & $(0.08)$ & $(0.21)$ & $(0.14)$ & $(0.52)$ & $(0.60)$ & $(0.10)$ \\
Brazil & 0.22 & $0.57^{*}$ & & $0.73^{*}$ & $0.52^{*}$ & $0.51^{*}$ & $0.62^{*}$ & $0.43^{* *}$ \\
& $(0.39)$ & $(0.02)$ & & $(0.0014)$ & $(0.039)$ & $(0.043)$ & $(0.011)$ & $(0.10)$ \\
Chile & $0.41^{* *}$ & 0.20 & 0.16 & 0.12 & 0.19 & 0.15 & 0.24 & 0.20 \\
& $(0.10)$ & $(0.44)$ & $(0.53)$ & $(0.66)$ & $(0.48)$ & $(0.58)$ & $(0.38)$ & $(0.45)$ \\
Paraguay & 0.075 & 0.09 & 0.30 & 0.39 & $0.59^{*}$ & 0.30 & $0.46^{* *}$ & 0.26 \\
& $(0.77)$ & $(0.74)$ & $(0.25)$ & $(0.14)$ & $(0.017)$ & $(0.26)$ & $(0.07)$ & $(0.32)$ \\
Uruguay & -0.004 & 0.10 & 0.29 & $0.49^{* *}$ & 0.12 & 0.12 & $0.45^{* *}$ & 0.36 \\
& $(0.99)$ & $(0.69)$ & $(0.26)$ & $(0.06)$ & $(0.66)$ & $(0.66)$ & $(0.08)$ & $(0.18)$ \\
\hline
\end{tabular}

Source: World Economic Outlook.

Magnitudes in parentheses indicate the probability of zero correlation.

$*$ and $* *$ denote significance at the five and ten percent levels.

Table 17b. Co-Movements in Real GDP Growth Sample Period: 1987-2006

\begin{tabular}{|c|c|c|c|c|c|c|c|c|}
\hline $\begin{array}{l}\text { Mercosur } \\
\text { Group }\end{array}$ & U.S. & EU & Brazil & $\begin{array}{l}\text { Andean } \\
\text {-Self }\end{array}$ & Caribbean & $\begin{array}{l}\text { Central } \\
\text { America }\end{array}$ & $\begin{array}{l}\text { LAC7 } \\
\text {-Self }\end{array}$ & $\begin{array}{l}\text { Mercosur } \\
\text {-Self }\end{array}$ \\
\hline Argentina & $\begin{array}{c}0.12 \\
(0.61)\end{array}$ & $\begin{array}{l}-0.38 \\
(0.10)\end{array}$ & $\begin{array}{c}0.12 \\
(0.60)\end{array}$ & $\begin{array}{c}0.62 * \\
(0.004)\end{array}$ & $\begin{array}{c}0.16 \\
(0.53)\end{array}$ & $\begin{array}{c}0.53 * \\
(0.019)\end{array}$ & $\begin{array}{c}0.77 * \\
(0.0001)\end{array}$ & $\begin{array}{c}0.18 \\
(0.46)\end{array}$ \\
\hline Brazil & $\begin{array}{c}0.28 \\
(0.23)\end{array}$ & $\begin{array}{c}0.05 \\
(0.84)\end{array}$ & & $\begin{array}{l}0.40 * * \\
(0.087)\end{array}$ & $\begin{array}{c}0.25 \\
(0.31)\end{array}$ & $\begin{array}{c}0.04 \\
(0.87)\end{array}$ & $\begin{array}{c}0.21 \\
(0.38)\end{array}$ & $\begin{array}{c}0.18 \\
(0.47)\end{array}$ \\
\hline Chile & $\begin{array}{l}-0.005 \\
(0.98)\end{array}$ & $\begin{array}{l}-0.096 \\
(0.69)\end{array}$ & $\begin{array}{c}0.21 \\
(0.38)\end{array}$ & $\begin{array}{c}0.17 \\
(0.48)\end{array}$ & $\begin{array}{l}-0.43^{*} \\
(0.07)\end{array}$ & $\begin{array}{l}0.044 \\
(0.86)\end{array}$ & $\begin{array}{c}0.23 \\
(0.35)\end{array}$ & $\begin{array}{c}0.31 \\
(0.20)\end{array}$ \\
\hline Paraguay & $\begin{array}{l}-0.027 \\
(0.91)\end{array}$ & $\begin{array}{l}-0.042 \\
(0.86)\end{array}$ & $\begin{array}{c}0.09 \\
(0.71)\end{array}$ & $\begin{array}{c}0.12 \\
(0.61)\end{array}$ & $\begin{array}{l}-0.20 \\
(0.41)\end{array}$ & $\begin{array}{l}-0.20 \\
(0.41)\end{array}$ & $\begin{array}{c}0.08 \\
(0.74)\end{array}$ & $\begin{array}{c}0.28 \\
(0.24)\end{array}$ \\
\hline Uruguay & $\begin{array}{l}0.40 * * \\
(0.08)\end{array}$ & $\begin{array}{l}-0.025 \\
(0.92)\end{array}$ & $\begin{array}{c}0.23 \\
(0.33)\end{array}$ & $\begin{array}{l}0.46^{*} \\
(0.05)\end{array}$ & $\begin{array}{c}0.06 \\
(0.81)\end{array}$ & $\begin{array}{c}0.37 \\
(0.12)\end{array}$ & $\begin{array}{c}0.65^{*} \\
(0.002)\end{array}$ & $\begin{array}{c}0.85^{*} \\
(0.0001)\end{array}$ \\
\hline
\end{tabular}

Source: World Economic Outlook.

partners or regional blocks on real growth in Latin America ${ }^{19}$ Openness reflects positively on real growth, implying a direct stimulus of real growth through the

\footnotetext{
${ }^{19}$ Real growth increases with an increase in openness relative to the U.S. in Grenada and St. Kitts. Real growth increases with an increase in openness, relative to Brazil, in Argentina. Real growth increases with an increase in openness, relative to the EU, in Colombia, Dominica, Grenada, St. Lucia, and St. Vincent. Real growth increases with an increase in openness, relative to the Andean group, in Brazil and El Salvador. Real growth increases with an increase in openness, relative to Central America, in Argentina, Chile, Costa Rica, Ecuador, Paraguay, and Trinidad and Tobago. Real growth increases with an increase in openness, relative to the Caribbean, in St. Kitts and Nevis. Real growth increases with an increase in openness relative to LAC7 in Chile, Ecuador, Guyana, St. Kitts, and Venezuela. Real growth increases with an increase in openness relative to Mercosur in Brazil.
} 
Table 18a. Co-Movements in Real GDP Growth Sample Period: 1987-2006

\begin{tabular}{lcccccccc}
\hline $\begin{array}{l}\text { Group of } \\
\text { Mercosur }\end{array}$ & U.S. & EU & Brazil & $\begin{array}{c}\text { Andean } \\
\text {-Self }\end{array}$ & Caribbean & $\begin{array}{c}\text { Central } \\
\text { America }\end{array}$ & $\begin{array}{c}\text { LAC7 } \\
\text {-Self }\end{array}$ & $\begin{array}{c}\text { Mercosur } \\
\text {-Self }\end{array}$ \\
\hline Argentina & -0.048 & 0.19 & 0.021 & -0.22 & $0.53^{*}$ & -0.02 & 0.28 & $0.47^{*}$ \\
& $(0.84)$ & $(0.43)$ & $(0.93)$ & $(0.37)$ & $(0.02)$ & $(0.93)$ & $(0.24)$ & $(0.04)$ \\
Brazil & 0.12 & -0.043 & & 0.37 & 0.22 & $0.49^{*}$ & -0.094 & -0.064 \\
& $(0.61)$ & $(0.86)$ & & $(0.12)$ & $(0.37)$ & $(0.03)$ & $(0.70)$ & $(0.79)$ \\
Chile & -0.054 & 0.049 & 0.17 & 0.22 & 0.20 & 0.25 & $0.80^{*}$ & 0.28 \\
& $(0.82)$ & $(0.84)$ & $(0.47)$ & $(0.37)$ & $(0.40)$ & $(0.30)$ & $(0.0001)$ & $(0.24)$ \\
Paraguay & -0.14 & -0.098 & 0.072 & -0.26 & $0.46^{*}$ & -0.34 & 0.28 & 0.13 \\
& $(0.56)$ & $(0.68)$ & $(0.76)$ & $(0.29)$ & $(0.04)$ & $(0.16)$ & $(0.24)$ & $(0.59)$ \\
Uruguay & $0.41 * *$ & -0.022 & 0.08 & $-0.52^{*}$ & $0.42^{* *}$ & -0.19 & 0.23 & 0.28 \\
& $(0.07)$ & $(0.93)$ & $(0.73)$ & $(0.02)$ & $(0.07)$ & $(0.44)$ & $(0.33)$ & $(0.25)$ \\
\hline
\end{tabular}

Source: World Economic Outlook.

Magnitudes in parentheses indicate the probability of zero correlation.

$*$ and $* *$ denote significance at the five and ten percent levels.

Table 18b. Average Shares of Trade ((Exports + Imports)/GDP) with Major Trading Partners (in percent) Sample Period: 1987-2006

\begin{tabular}{lcccccccc}
\hline $\begin{array}{l}\text { Group of } \\
\text { Mercosur }\end{array}$ & U.S. & EU & Brazil & $\begin{array}{c}\text { Andean } \\
\text {-Self }\end{array}$ & Caribbean & $\begin{array}{c}\text { Central } \\
\text { America }\end{array}$ & $\begin{array}{c}\text { LAC7 } \\
\text {-Self }\end{array}$ & $\begin{array}{c}\text { Mercosur } \\
\text {-Self }\end{array}$ \\
\hline Argentina & 2.76 & 4.54 & 0.18 & 0.73 & 0.067 & 0.082 & 6.12 & 6.05 \\
Brazil & 3.9 & 4.85 & & 0.6 & 0.12 & 0.09 & 2.69 & 2.32 \\
Chile & 8.76 & 11.7 & 3.33 & 2.71 & 0.21 & 0.24 & 9.86 & 6.99 \\
Paraguay & 4.29 & 6.57 & 13.66 & 0.62 & 0.28 & 0.32 & 22.7 & 24.2 \\
Uruguay & 3.26 & 6.58 & 7.3 & 0.82 & 0.08 & 0.12 & 14.5 & 13.4 \\
\hline
\end{tabular}

Source: Direction of Trade Statistics.

export channel. In addition, imports may provide an additional stimulus that contributes to real growth. In general, the evidence spells out limited stimulus effects of increased openness on developments in real growth over time in Latin America.

To formalize the relationship between trade linkages and co-movement in real output growth, Table 19a presents the results of cross-country regressions in the sample period $1970-1986 .{ }^{20}$ The dependent variable is the correlation coefficient between real growth in a given country with real growth in the remainder of its

\footnotetext{
${ }^{20}$ Results are robust in experiments that instrument the trade shares with lagged growth rates in the respective countries to account for potential endogeneity, or follow a weighted least squares regression where variables are weighted by the inverse of the standard error of the time-series distribution. That is variables that have high variance are weighted less heavily in the cross-section regression.
} 
Table 19a. The Impact of Trade Linkages on Co-Movements in Real GDP Growth across Countries

\begin{tabular}{lcccc}
\hline & \multicolumn{4}{c}{ Explanatory Variables } \\
\cline { 2 - 5 } $\begin{array}{l}\text { Dependent Variable Corre- } \\
\text { lations in Real Growth 1/ }\end{array}$ & Constant & $\begin{array}{c}\text { Average Share of } \\
\text { Trade/GDP }\end{array}$ & Constant & $\begin{array}{c}\text { Average Share of } \\
\text { Trade/GDP }\end{array}$ \\
\cline { 2 - 5 } & \multicolumn{2}{c}{$1970-1986$} & $0.11^{*}$ & 0.00066 \\
\hline 1. Across all Countries & $0.32^{*}$ & $-0.001^{* *}$ & $(5.72)$ & $(0.80)$ \\
(243 obs.) & $(17.97)$ & $(-1.71)$ & 0.052 & 0.003 \\
2. With the U.S. (27 obs.) & $0.16^{*}$ & $-0.0004^{*}$ & $(0.77)$ & $(1.67)$ \\
& $(3.05)$ & $(-0.47)$ & -0.097 & $0.043^{* *}$ \\
3. With the EU (27 obs.) & $0.39^{*}$ & -0.0014 & $(-1.58)$ & $(1.89)$ \\
& $(6.64)$ & $(-0.64)$ & $0.18^{*}$ & -0.013 \\
4. With Brazil (26 obs.) & $0.36^{*}$ & -0.018 & $(2.89)$ & $(0.85)$ \\
& $(6.64)$ & $(-0.58)$ & $0.18^{*}$ & -0.004 \\
5. With Andean (27 obs.) & $0.37^{*}$ & -0.0009 & $(3.30)$ & $(-0.77)$ \\
& $(8.79)$ & $(-0.78)$ & 0.065 & -0.0028 \\
6. With Caribbean (27 obs.) & $0.32^{*}$ & $-0.0067^{* *}$ & $(1.10)$ & $(-0.88)$ \\
7. With Central America $(27$ & $(5.32)$ & $(-1.77)$ & 0.065 & -0.02 \\
obs.) & $0.33^{*}$ & $-0.0067^{* *}$ & $(1.10)$ & $(1.46)$ \\
8. With LAC7 (27 obs.) & $(6.13)$ & $(-1.77)$ & $0.24^{*}$ & -0.0002 \\
& $0.40^{*}$ & 0.00034 & $(3.82)$ & $(-0.06)$ \\
9. With Mercosur (27 obs.) & $(8.44)$ & $(0.29)$ & $0.21^{*}$ & 0.01 \\
& $0.38^{*}$ & $0.00015^{*}$ & $(3.25)$ & $(1.08)$ \\
\hline
\end{tabular}

1/ Correlations are between real growth in Latin America with real growth in common sources (U.S., Brazil and the EU) and regional blocks (Andean, Caribbean, Central America, LAC7 and Mercosur).

Table 19b. The Impact of Trade Linkages on Co-Movements in Real GDP Growth across Countries: 1987-2006

\begin{tabular}{lccc}
\hline \multirow{2}{*}{$\begin{array}{l}\text { Dependent Variable } \\
\text { Correlations in Real }\end{array}$} & \multicolumn{3}{c}{ Explanatory Variables } \\
\cline { 2 - 4 } Growth 1/ & Constant & $\begin{array}{c}\text { Average Share of Trade/ } \\
\text { GDP }\end{array}$ & $\begin{array}{c}\text { Correlation in start-up } \\
\text { Deposit lnterest Rate }\end{array}$ \\
\hline 1. Across all Countries & $0.072^{*}$ & 0.0018 & $0.076^{*}$ \\
(967 obs.) & $(7.74)$ & $(1.17)$ & $(2.53)$ \\
2. With the Caribbean $(409$ & $0.028^{*}$ & -0.0034 & $0.11^{*}$ \\
obs.) & $(1.88)$ & $(-0.41)$ & $(2.27)$ \\
\hline
\end{tabular}

1/ Correlations are bilateral between all country pairs in Latin America and with common sources (U.S. and the EU).

regional block, other regional blocks, or the common sources. The independent variable is the corresponding trade linkage, the average trade share with the relevant partner(s) over the same time span.

Higher trade linkages increase specialization based on comparative advantages across countries, decreasing co-movements in real output growth across countries 
of Latin America. Regression 1 presents the results of the relation between comovements in real growth and the corresponding trade linkages across all countries with all common sources and regional blocks in Latin America (243 observations). The coefficient is negative and statistically significant.

Higher trade linkage decreases co-movements in real output growth across countries in Latin America with Caribbean growth, as evident in Regression 5. In the remaining regressions, the coefficients measuring the relationship between output co-movement and trade shares are statistically insignificant.

There is an increase in co-movement in real growth across Latin American countries in response to higher trade linkages to the EU. The right-hand side panel of Table 19a presents the results of cross-country regressions that measure the effects of trade linkages on co-movements in real output growth across countries in the sample period 1987-2007.

Overall, the effect of trade linkages on co-movements in real growth across countries appears mixed. The bulk of the evidence indicates insignificant effects. In 1970-86, the significant evidence indicates a reduction in business-cycle synchronization with trade linkages. Stronger trade linkages are associated with increased inter-industry specialization across countries. As industry-specific shocks become more dominant, the spillover effects of trade linkages are weaker across countries, decreasing co-movements of cycles. In contrast, the significant evidence in 1987-2007 indicates greater synchronization of co-movements in real growth with trade linkages. More trade relations, particularly with the EU, increase the probability of transferring shocks across countries, increasing growth comovements.

\section{B. Conditional dynamics}

Contemporaneous correlations may be highly affected by random noise that disguises a robust relationship in the steady state. The above analysis employs correlation coefficients to measure co-movements in real growth across countries. Contemporaneous correlations do not capture, however, the dynamics implied by persistence in own and cross relations. To address this shortcoming, the following empirical model is estimated:

$$
D y_{i t}=\alpha_{0}+\alpha_{1} D y_{i t-1}+\alpha_{1} D y_{i t-2}+\alpha_{3} D y_{j t}+\alpha_{4} D y_{j t-1}+\alpha_{5} D y_{j t-2}
$$

Here, $D y_{i t}$ is real growth in country $i$ at time $t . D y_{j t}$ is real growth in the common source or regional block. Co-movements in real growth is measured by $\sum_{i=3}^{n} \alpha_{i}$ 
where $n$ is the maximum length of significant lags, i.e., $i=3$, 4, or 5 .

Co-movement in real growth varies negatively with trade openness. Using this measure of co-movements in real growth as a dependent variable in cross-country regressions, following the format in Table 19, the results indicate the impact of openness on conditional co-movements over time. Across all countries, comovement in real growth varies negatively and significantly with trade openness with a coefficient -0.23 (t-ratio -1.76) in the sample period 1970-86. In the latter sample period, co-movements in real growth vary negatively and significantly with trade openness with a coefficient -0.39 (t-ratio -1.82) in the sample period 1987$2007 . .^{21}$

The results provide further support to the effect of trade integration on divergence in business cycles across Latin America, and with the common sources (Brazil, U.S., and EU). As countries become more specialized in trade, comovements in business cycles at the aggregate level are less evident.

\section{Bilateral co-movements and financial linkages}

It is possible that grouping of regional blocks may have distorted the effect of trade openness on co-movements in real growth. To further verify the robustness of the above evidence, this extension considers the effect of bilateral trade relations on co-movements in real growth for country pairs in Latin America and with the common sources (968 observations). While co-movement in real growth is symmetric for country pairs, measures of trade openness are not symmetric for the same pair.

Regressing correlations in real growth on trade openness across countries yields positive, although statistically insignificant, coefficients in the sample periods 1970-86 and 1987-2007. The results provide further evidence that discounts the relevance of trade relations to synchronization of business cycles in Latin America.

The results support a more important role for financial linkage in determining co-movement in business cycles. ${ }^{22}$ Data are available to measure co-movements in bilateral short-term deposit interest rates across countries of Latin America and with the U.S. and the EU in the sample period 1987-2007. Upon introducing comovements in short-term interest rates into the cross-country regression (see Table

\footnotetext{
${ }^{21}$ Detailed results are available upon request.

${ }^{22}$ Average trade shares vary positively and significantly with correlations in short-term interest rates between individual countries in Latin America and counterparts in the following regional blocks: Caribbean, Mercsour, and Central America.
} 
19b), interest rate movements help synchronize business cycles in a sample that combines all countries in Latin America and the common sources. Interest rate comovements are also significant in determining co-movements in real output growth in a sub-sample that includes co-movements in business cycles in Latin America with Caribbean countries.

\section{Summary and Conclusion}

The paper studies co-movements in business cycles using real GDP growth in various countries of Latin America. Countries belong to several regional blocks: Andean, Caribbean, Central America, LAC7, and Mercosur, with some overlap in membership across some groups. Real growth in each member country is assumed to vary with common sources, other countries within its regional block, and other regional blocks in Latin America. Common sources include the U.S., the EU, and a major regional source, Brazil.

The paper presents evidence on co-movements across the various regional blocks and with the major common sources. To track possible evolution in these co-movements over time, the sample period 1970-2007 is truncated into two subsamples: 1970-86 and 1987-2007. The latter period has witnessed more efforts to integrate the global economy by strengthening financial and trade linkages. The shares of trade (exports plus imports) to GDP for each country in Latin America approximate trade linkages with the remaining countries within its regional block, other regional blocks, and the common sources. Financial linkages are measured by co-movement in short-term deposit interest rate in the specific Latin country with other counterparts.

Despite increased efforts for integration and higher trade linkages, comovements in real GDP growth appear, in general, less significant across Latin America and with the common sources in 1987-2007, compared to 1970-1986. Further, trade linkages do not appear to track significant co-movements in real growth across Latin America and with the common sources.

Trade provides each country the opportunity to maximize on its comparative advantage, resulting in more specialization such that industry-specific shocks decrease business-cycle synchronization at the macro level. To formalize the relationship, correlation between real growths is regressed on the corresponding measure of trade linkage for each country with the common sources or regional blocks. The analysis employs all correlations across countries, and across 
subgroups that measure correlations with a specific common source or a regional block. The significant results indicate a negative effect of trade linkages on comovements in real growth in 1970-86.

More recently, in 1987-2007, an increase in trade linkages, with the EU, is consistent with an increase in business-cycle synchronization. Shocks are transferred through the trade channel, increasing the spill-over of EU shocks across countries of Latin America. The bulk of the evidence remains, however, insignificant, ruling out the effect of trade linkage in explaining business-cycle fluctuations across countries, in general.

Using bilateral country pairs, the evidence supports the importance of financial linkage to increase business-cycle synchronization across all countries of Latin America and with the common sources in the U.S., Brazil, and the EU. The evidence is based on a test of the effects of financial linkage, as measured by correlations in short-term deposit interest rates, on co-movements in real output growth during the period 1987-2007. Interest rate correlations are also an important determinant of comovement in Caribbean business cycles with real growth across countries of Latin America.

The results provide motivation for future research. Some of the findings regarding significant, or lack of, correlations present a puzzle in the context of trade relations, regional interdependence and common perceptions. Moreover, the robust evidence of the negative effects of trade linkages on business-cycle synchronization challenges some of the earlier findings arguing for the opposite effects of trade linkages. In general, the evidence establishes that output comovements could be far more complex than what trade linkage might explain.

Received 15 March 2011, Revised 09 May 2011, Accepted 16 May 2011

\section{References}

Baxter, M. and M. Kouparitsas. (2004), "Determinants of Business Cycle Co-movement: A Robust Analysis," NBER Working Paper No. 10725, (Cambridge: Massachusetts). Cardorelli, R. and M. A. Kose. (2004), "Economic Integration, Business Cycle, and Productivity in North America," IMF Working Paper, WP/04/138.

Clark, Todd E., and van Wincoop, Eric. (2001), "Borders and Business Cycles," Journal of International Economics, 5, pp. 59-85.

Doyle, B. and J. Faust. (2003), "Break in the Variability and Co-movement of G-7 Economic Growth," Working Paper, Federal Reserve Board. 
Frankel, Jeffrey A., and Rose, Andrew K. (1998), "The Endogeneity of the Optimum Currency Area Criteria," Economic Journal, 108, pp. 1009-25.

Heathcote, Jonathan, and Fabrizio Perri. (2002), "Financial Autarky and International Business Cycles," Journal of Monetary Economics, 49, pp. 601-28.

Helbling, T. and T. Bayoumi. (2002), "G-7 Business Cycle Linkages Revisited," IMF Working paper (Washington: International Monetary Fund).

Gregory, A. W., A. C. Head, and J. Raynauld. (1997), "Measuring World Business Cycles," International Economic Review, 38, pp. 677-702.

Kalemli-Ozcan, S., B. Sorensen, and O. Yosha. (2003), "Risk Sharing and Industrial Specialization: Regional and International Evidence," American Economic Review, 93, pp. 903-18.

Knight, W. E. (1966), “A Computer Method for Calculating Kendall's Tau with Ungrouped Data," Journal of the American Statistical Association, 61, pp. 436-439.

Kose, M. A., C. Otrok, and C. Whiteman. (2003), "International Business Cycles: World Region, and Country Specific Factors," American Economic Review, 93, pp. 121639.

Kose, M. A., E. S. Prasad, and M. Terrones. (2003), "How Does Globalization Affect the Synchronization of Business Cycles?" American Economic Review-Papers and Proceedings, 93, pp. 57-62.

Kose, M. A., and K. Yi. (2005), "Can the Standard International Business Cycle Model Explain the Relation between Trade and Co-movement?" Journal of International Economics.

Kose, M. A., C. Otrok, and C. Whiteman. (2005), "Understanding the Evolution of World Business Cycles," IMF Working paper, WP/05/211.

Imbs, J. (2004a), "Trade, Finance, Specialization and Synchronization," Review of Economics and Statistics.

Imbs, J. (2004b), "Real Effects of Financial Integration," IMF Staff papers (Washington: International Monetary Fund).

Nelson, Charles R., and Charles I. Plosser. (1982), "Trends and Random Walks in Macroeconomic Time Series," Journal of Monetary Economics, 10, September, pp. 139-62.

Noether, G. E. (1967), Elements of Nonparameteric Statistics, New York: John Wiley and Sons.

Otto, Glenn; Voss, Graham, and Luke Willard. (2003), "Understanding OECD Output Correlations," Working Paper, University of New South Wales.

Stock, James H. and Mark W. Watson. (2003), "Understanding Changes in International Business Cycles,” NBER Working Paper No. 9859 (Cambridge: Massachusetts). 


\section{Appendix}

\section{A. Data sources}

Annual data are as follows:

1. Real Output: gross domestic product, constant prices, WEO, WNGDP.

2. Price Level: the deflator for GDP, WEO, NGDP_D.

3. Government Spending: nominal values of all payments by the government.

4. Money Supply: broad money, WEO, WMB.

5. Private Consumption: current prices of private consumption expenditure in national currency, WEO, WNCP.

6. Private Investment: current prices of private investment expenditure in national currency, WEO, WNFIP.

7. Exports of Goods and Services: current prices, WEO, WNX.

8. Imports of Goods and Services: current prices, WEO, WNM.

9. Exchange Rate: real effective exchange rate, INS.

10. Interest Rate: Deposit rate, IFS, 60L.zF., Discount rate, IFS, 60..zF.

Sources: World Economic Outlook (WEO), Information Notice System (INS), and International Financial Statistics (IFS) data baks available from the International Monetary Fund, Washington D.C. 